Published in Transportation research Part b: methodological, 2018, vol. 111, pp. 19-38, which should be cited to refer to this work.

DOI : $10.1016 / j . t r b .2018 .03 .002$

\title{
Train Timetable Design Under Elastic Passenger Demand
}

\author{
Tomáš Robenek * Shadi Sharif Azadeh $* \dagger \quad$ Yousef Maknoon $* \ddagger$ \\ Matthieu de Lapparent* $\quad$ Michel Bierlaire*
}

March 1, 2018

*Transport and Mobility Laboratory (TRANSP-OR), School of Architecture, Civil and Environmental Engineering (ENAC), École Polytechnique Fédérale de Lausanne (EPFL), CH-1015 Lausanne, Switzerland, \{tomas.robenek, shadi.sharifazadeh, yousef.maknoon, matthieu.delapparent, michel.bierlaire\}@epfl.ch

${ }^{\dagger}$ Econometric Institute, Erasmus University Rotterdam, P.O. Box 1738, 3000 DR, Rotterdam, Netherlands

${ }^{*}$ Faculty of Technology, Policy and Management, Delft University of Technology, 2628 BX, Delft, Netherlands

${ }^{\S}$ University of Applied Sciences and Arts Western Switzerland (HES-SO). School of Business and Engineering Vaud (HEIG-VD). Avenue des sports 20 - CP 521 - 1401 Yverdon-les-Bains, Switzerland 


\begin{abstract}
A passenger centric timetable is such a timetable that the satisfaction of the passengers is maximized. However, these timetables only maximize the probability of a passenger to take the train, but provide no insight on the actual choices of the passengers. Therefore, in this manuscript we replace the deterministic passenger satisfaction function with a probabilistic demand forecasting model inside of the passenger centric train timetable design. The actual forecasts lead to a realistic train occupation. Knowing the train occupation, we can estimate the revenue and to use pricing as a mobility management to further improve the level-of-service. We use a logit model that we calibrate to reflect the known demand elasticities. We further include a competing operator as an opt-out option for the passengers. Subsequently, we integrate the passenger centric train timetabling problem with a ticket pricing problem. We solve the elastic passenger centric train timetabling problem for various types of timetables using a simulated annealing heuristic on a case study of Israeli Railways. The results of our case study show that the generated revenues can be increased by up to $15 \%$ when taking into account the passengers' behavior along with a specific pricing scheme. This study further confirms the advantages of hybrid cyclicity.
\end{abstract}

Keywords: Passenger Centric Train Timetabling Problem, Railway Demand Forecasting, Hybrid Cyclicity, Ticket Pricing, Revenue 


\section{Introduction}

From economic theory, it is well known that the demand is influenced by the supply. In the railway context: different timetables would attract different passengers, as they provide different levels of services. Therefore, it is important to include the passenger demand and its elasticity in the timetable design. This is particularly crucial nowadays, when the railway market is open for competition. This has lead to a release of the government's subsidies and the operators thus face a pressure to be profitable by increasing their revenues.

The passenger demand itself can be predicted through the choices that the passengers face: mode, route, operator, service and departure time. In reality, the passengers make an integrated decision that is split into the 4-step procedure due to its complexity. At first, the passengers decide on their mode of transport, i.e. car, bus, train, etc. Subsequently, they decide on their exact path within the selected mode, e.g. the exact trains and interchanges. As transportation is offered by several providers, the passengers can select which one to take and in what kind of service, e.g. first class with a private operator, having a club card of a specific operator, etc. Lastly, the departure time choice of the passengers is affected by the trip purpose (commuting to be on time at work, leisure to be on time for cinema for instance, etc.). This choice is the so called time dependency of the demand. The mode choice and the operator choice are especially important when optimizing a service. If not included, the passengers are captive in the system and such optimization would lead to unrealistic performance (i.e. departure times leading to a lower ridership than anticipated).

A train timetable is defined as a set of arrival and departure times of each train from each of its stopping stations and it is the output of the Train Timetabling Problem (TTP). Typically, the TTP models use the simplifying assumption that the passengers always take their shortest paths (see Caprara et al. (2002) for instance) and omit the demand from the problem. The issue of this approach is that being a shortest path is dependent on the actual departure time. Therefore, recent models relax this assumption and include the demand in the optimization (see Schmidt and Schöbel (2015) for instance). However, these models only increase the attractiveness of a timetable and cannot estimate the realized demand and the underlying revenue. In order to do so, a demand forecasting model is needed. One of such models, integrating the train timetabling and the demand forecast, is presented by Cordone and Redaelli (2011). However, they only consider the mode choice and omit the other choices. Moreover, they consider the timetable only as a single cycle within a cyclic timetable. In the model of EspinosaAranda et al. (2015), the mode choice is combined with a departure time choice. But their application involves a single high speed railway line and lacks the network dimension (i.e. the route choice).

In this paper, we extend and adjust the Passenger Centric Train Timetabling Problem (proposed by Robenek et al. (2016)), that can design a timetable for a whole railway network, with a demand forecasting model. We denote the new model as the Elastic Passenger Centric Train Timetabling Problem (EPCTTP). The objective of the new optimization framework is to maximize the Train Operating Company's (TOC) revenue. The framework is using a discrete choice model to predict the demand throughout the timetable design process. We assume prior solving of the mode choice model and we solve the route choice model along with the departure time choice and the operator choice. The following attributes have influence on the passengers' choices: the travel time, the desired arrival time to their destinations (the time dependency), the ticket fare and the capacity of the trains. We include 
a universal option of opting out into the passengers' choice set(s), in order to avoid their captivity. The demand elasticity and other parameters of the discrete choice model are calibrated to known values from the literature and provide a "ready to use" framework. It can design cyclic, non-cyclic and hybrid cyclic timetables. The resulted timetables increase the ridership through accounting for the passengers' wishes and therefore, they increase the operator's revenue as well. We test this approach on a case study of Israeli Railways.

In addition to the above, we further relax the ticket fare to be a decision variable. Such approach leads to a better utilization of the TOC's fleet and to a further increase in its revenue: shift passengers from overcrowded trains to under-used trains, attract back the passengers, who would otherwise opt-out, etc. The resulting framework combines pricing problem with an integrated demand-supply timetable design. It differs from the existing literature, where the pricing and revenue management is performed on a fixed timetable.

The manuscript is structured as follows: Section 2 presents a survey of the literature, depicting the demand interaction in the current timetabling models, along with the literature on railway demand forecasting as well as pricing and revenue management in railways. Subsequently, we propose a demand forecasting model in Section 3, that is further incorporated into a train timetable optimization problem described informally in Section 4. The insight, about the methodology of how to solve such integrated demand-supply optimization framework, is given in Section 5. The benefits and the impacts of the framework are shown on a case study of Israeli Railways in Section 6. We finalize the paper by drawing some conclusions and discussion of possible extensions in Section 7.

\section{Literature Review}

Since the aim of this study is to introduce the demand and its elasticity into the train timetabling, the focus of our literature review is on the passenger demand representation in the railway planning literature. At first, we present the demand representations used in the train timetabling problems (Section 2.1) and continue with the various demand forecasting techniques used in the railway context (Section 2.2). Lastly, we discuss how some of the forecasting techniques are used in the revenue management and ticket pricing within the railways (Section 2.3).

\subsection{Demand Representations}

The most basic representation of the railway demand is an Origin-Destination (OD) matrix. Such a matrix is typically used in the Line Planning Problem (LPP) to determine the frequency of a train line (Schöbel (2012)). However, one of the recent models by Canca, Barrena, De-Los-Santos and Andrade-Pineda (2016) considers the actual paths of the passengers while minimizing their travel time.

Once the lines are designed, the Train Timetabling Problem (TTP) assigns a departure time to each train subject to the operational constraints. Two versions of this problem exist: cyclic and non-cyclic. In the cyclic TTP (Peeters (2003),Zhou et al. (2017), Sparing and Goverde (2017), for instance), the main focus is on the cyclicity constraints, whereas in the non-cyclic TTP (Caprara et al. (2002)) 
the departure times do not have to follow any specific pattern. Traditionally, both problems assume that the passengers follow the shortest path. Therefore, there is no need to include the route choice dimension explicitly and the optimization is performed on the attributes of the shortest paths between the ODs.

Recently, this assumption has been relaxed and the passengers can choose from several paths while minimizing their total travel time: refer to Hoppmann et al. (2015) for the cyclic TTP and Schmidt and Schöbel (2015) for the non-cyclic TTP. However, this approach can be further improved by maximizing the passenger satisfaction instead. The passenger satisfaction better reflects the human behavior. It combines the in-vehicle-time, the waiting time, the number of transfers and the desired arrival time to the destination of a given path, each weighted by the respective human perception. Such approach is called the Passenger Centric Train Timetabling Problem and it can design both: cyclic and non-cyclic timetables (Robenek et al. (2016)) or a recently proposed hybrid cyclic timetable by Robenek et al. (2017) (for a further description of this timetable refer to Section 4).

A different approach is to use the passenger arrival rates to their origin stations, in order to design a timetable (Luethi et al. (2007), Niu and Zhou (2013), Barrena et al. (2014a,b), Sun et al. (2014), Wang et al. (2015), Yin et al. (2016, 2017)). Similarly, Canca et al. (2014) use the analytical approach based on the passenger arrival rates. However, this method is only suitable for high frequency services such as the public transit.

\subsection{Demand Forecasting}

In order to forecast the demand, its behavior in form of the decisions needs to be modeled. As mentioned before, the main decisions are: mode, route, operator, service and departure time choice. One of the first forecasting models has been introduced by Wardman (1997). He shows a direct demand model taking into account the mode competition (between rail and car) and the service offer (cost and service quality). He further extends this model with additional attributes such as car ownership, car travel time and fuel cost in Wardman (2006). Other models are investigated by Wardman et al. (2007). The main focus is on the generalized cost, i.e. service choice. All of these models are in the settings of the British railway market.

Another group of demand forecasting models focuses on high speed rail. The case studies involve Italy (integrated demand model combining demand growth, mode choice and induced demand by Ben-Akiva et al. (2010)), China (hybrid approach combining empirical mode decomposition and gray support vector machine by Jiang et al. (2014)) and Sweden (nested logit model for mode choice by Börjesson (2014)), among others. A recent survey on a high speed railway demand forecasting models is presented by Börjesson (2014).

Lastly, Canca, De-Los-Santos, Laporte and Mesa (2016) use mode choice based on the generalized cost in order to integrate and to improve the network design with line planning.

Remark All above models only forecast the demand and do not combine it with a timetabling problem. The supply-demand interaction is a fairly new topic in the timetabling context. Two applications of a train timetabling problem incorporating a mode choice forecast exist: Cordone and Redaelli (2011) and Espinosa-Aranda et al. (2015). Cordone and Redaelli (2011) maximize the demand cap- 
tured by a cyclic timetable using a logit model as a mode choice and Espinosa-Aranda et al. (2015) maximize the profit of a non-cyclic timetable using a constrained nested multinomial logit model as a mode choice combined with a departure time choice.

\subsection{Revenue Management and Pricing}

Typically, the demand forecasting models are used in a combination with the Revenue Management (RM) and pricing problems. The aim of these problems is to maximize the revenue while optimizing the prices based on the demand forecasts.

In the (railway) pricing problems, the strategy is to adjust the ticket prices based on a given timetable. The key attributes justifying the price variation are space (busy parts of the network vs. under-used parts of the network) and time of the day (peak hours vs. off-peak hours). One of the first frameworks to analyze different pricing policies was introduced by Nuzzolo et al. (2000). They present a nested logit model that takes into account elementary trains, instead of the frequencies. The model combines route choice, departure time choice and service choice. However, an empirical study of the effects of pricing policies and demand elasticities on a service choice in the context of Dutch Railways (van Vuuren (2002)) suggests that the peak hour demand is rather inelastic. This makes the strategy of higher prices during peak hours more effective than a price reduction during the off-peaks (Whelan and Johnson (2004)). This conclusion was found in the context of avoiding train overcrowding using an incremental logit model to do the forecasts. The model is predicting route choice, departure time choice and service choice. A similar goal, of flattening the demand throughout the service, is pursued by Li et al. (2006). They propose several pricing schemes combining spatial and time attributes. The passenger behavior is mimicked through activity-based microsimulation that takes into account mode choice, route choice and service choice.

The RM models, on the other hand, focus more on the price adjustments related to the demographics of the population. The key attributes justifying the price variation are the time of the purchase, which is related to the trip purpose (leisure travelers buy their tickets early vs. business travelers, who buy last minute tickets), class (1st class vs. 2nd class), and others. For a more detailed overview discussing the principal differences in RM of airlines and railways refer to Armstrong and Meissner (2010).

Overall, the literature on RM in railways is scarce and focuses solely on the price variation over the time of the purchase. A quantitative analysis on the pricing suggestions for Indian Railways is shown by Bharill and Rangaraj (2008) and in Hetrakul and Cirillo (2014), a multinomial logit and latent class models are tested within the pricing optimization framework. Both focus on service choice.

\subsection{Contribution}

In this paper, we present a model that combines train timetabling, ticket pricing and passenger assignment model. Our goal is to find the desirable departure time of the trains as well as their ticket price by using probabilistic passenger assignment model. We focus on double-track railway in a network with high passenger density. The problem has application in inter-city or regional railways. 


\section{Demand Model}

From the demand modeling perspective, the decision of travelers are hierarchically modeled as a mode choice and route choice. Figure (1) presents the steps of modeling travelers' demand.

In this problem, we assume that a mode choice model has been already solved to predict the railway market share. Therefore, the train operating company wishes to maximize its profit within the maximum potential demand that can be captured by the railway company. The potential demand can be either captured by the train operating company or its competitors. In the later case, either they use the service provided by the competitor or leave the system.

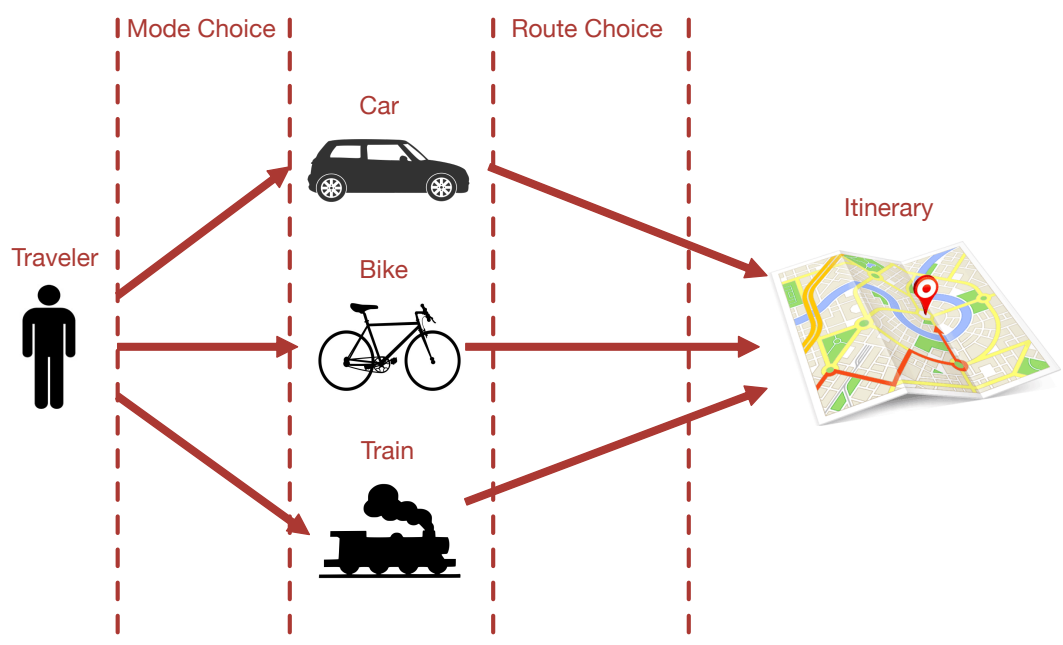

Figure 1: Decision process of a traveler

A passenger group is characterized by an Origin-Destination (OD) pair $i \in I$ and a desired arrival time $t \in T_{i}$ at destination. The actual value of the group's desired arrival time is represented by the parameter $a_{i t}$ in the set $T_{i}$ (in our model, time is discretized in minutes started from the mid-night as expressed as a set $\bar{T}=\{1, \ldots, H\}$ in which $H$ is the length of the planning horizon). Therefore, the combination of indices $(i, t)$ forms a unique group of passengers of size $n_{i t}$ (i.e. number of passengers in the group). For example, assume that there are 5 groups of passengers that wish to travel between the OD pair $i=1$. If travelers from the third group wishes to arrive at 8 a.m., the parameter $a_{13}$ is set to 480 .

Since we assume that the line planning problem has been solved a priori, a set of lines as well as their frequencies are known to the operator. Lines are defined by the set $\ell \in \mathrm{L}$. A line is an ordered sequence of stopping stations, that includes dwell times at the stations and travel time in between the stations. Each line also consists a set of segments $s \in S^{\ell}$. A segment is a part of infrastructure, where a train (line) does not stop. Finally, a train is defined by its line $\ell \in \mathrm{L}$ and its position within the sequence of available trains $v \in \mathrm{V}^{\ell}$ per line $\ell$.

For each OD pair $i$, first we determine the set of routes (we note it by $\hat{\mathrm{P}}_{i}$ ) as a combination of lines connecting the origin to the destination. Thereafter, a set of paths is defined as a product of routes and arrival times (i.e. $\mathrm{P}_{\mathrm{i}}=\widehat{\mathrm{P}}_{\mathrm{i}} \times \mathrm{T}$ ). Given the operational constraints (capacity) of the model this set 
might be reduced for specific groups: $P_{i t} \subseteq P_{i}$.

To model the number of passengers captured by train competitors, we include opt-out options in the set of paths $P_{i}$. Since we assume a prior solving of the mode choice model, a passenger can exit the system only by usage of another TOC. If a competing operator exists, it is sufficient to add his itineraries inside of the set $P_{i}$. However, since the existence of a competition is not yet wide spread, we propose the following assumption: given the nature of the railway competition (fighting for the same finite demand), the OD pairs served, the departure times and the prices of the competing operatos are typically very similar, i.e. the opt-out option for every passenger group is the current shortest path (that has the lowest value of the in-vehicle time and has zero waiting time in the transfer and arrives to the passengers' destination exactly at their desired arrival time) and its underlying ticket fare. Such an assumption is realistic and allows to explore the "worst" case scenario.

Consider the example, where going from point A to point B takes one hour with one change of ten minutes, using the shortest path. The opt-out option would be set to this time, therefore, the TOC cannot offer a much worse path, otherwise the passenger would leave the system through the opt-out option.

When faced with a set of of discrete path alternatives, it is assumed that a passenger chooses the one that yields her maximum level of utility (Ben-Akiva and Lerman (1985)). For a traveler in group $(i, t)$, utility $\mathcal{U}$ for a path $p \in P_{i t}$ is defined as:

$$
\mathcal{U}_{i t}^{p}=\mathcal{V}_{i t}^{p}+\varepsilon_{i t}^{p}
$$

The deterministic component $\mathcal{V}$ of the utility function is a function of observable attributes, i.e. relevant variables that describe a choice alternative and whose levels cause the expected level of utility. Note that we don't consider any source of individual heterogeneity outside of the error term $\varepsilon$. In our framework, only attributes of alternatives enter the utility function. For a group $(i, t)$, all passengers face the same levels of these attributes: $\mathcal{V}^{p}$ is only function of $(i, t)$. We also assume that $\mathcal{V}_{i t}^{p}$ is defined as a linear combination of the following travel attributes:

- in-vehicle-time $r_{i}^{p}-$ is the total time spent on board of trains in path $p$.

- waiting time $w_{i t}^{p}-$ is the total time passengers spend waiting in transferring stations.

- number of transfers $u^{p}$

- schedule passenger delay - indicates the time dependency of passenger demand and drives the departure time choice. Given the desired arrival time $a_{i t}$ to the destination of passenger group $(i, t)$, it can either be on time (equal to zero), early or late $\left(\bar{a}_{i t}\right.$ being the actual arrival time).

$$
\begin{aligned}
& -\operatorname{early}-\delta_{i t}^{p}=\max \left(a_{i t}-\bar{a}_{i t}, 0\right) \\
& \text { - late }-\gamma_{i t}^{p}=\max \left(0, \bar{a}_{i t}-a_{i t}\right)
\end{aligned}
$$

The deterministic component is therefore defined as follows: 


$$
\mathcal{V}_{i t}^{p}=-\beta_{V}^{\prime} \cdot r_{i}^{p}+\beta_{W}^{\prime} \cdot w_{i t}^{p}+\beta_{T}^{\prime} \cdot u^{p}+\beta_{E}^{\prime} \cdot \delta_{i t}^{p}+\beta_{L}^{\prime} \cdot \gamma_{i t}^{p}
$$

The parameters $\beta$ weigh the different attributes. They model sensitivity of the passengers to the change in the related attributes. Note that they are considered to be the same across individuals (homogeneity assumptions). The values of the $\beta$ s have to be estimated from data and have the following signs: $\beta_{\mathrm{V}}^{\prime}>0, \beta_{W}^{\prime}<0, \beta_{\mathrm{T}}^{\prime}<0, \beta_{\mathrm{E}}^{\prime}<0$, and $\beta_{\mathrm{L}}^{\prime}<0$. In this research, we use values of $\beta$ s from the literature. To do so, we rescale the utility function by $-\beta_{V}^{\prime}$ in order to convert the utility into travel time units. Indeed, the $\beta$ parameters themselves are not directly transferable, since they include the scale parameter of the error term. The scale parameter cancels out when ratios of parameters are considered, so the values of these ratios are comparable from one model to the next. The values of the parameters are as follows:

- $\beta_{\mathrm{W}}=\beta_{W}^{\prime} / \beta_{V}^{\prime}$ is the substitution rate between waiting time and in-vehicle-time per minute. Its value is equal to -2.5 (Wardman (2004)).

- $\beta_{\mathbf{T}}=\beta_{\mathrm{T}}^{\prime} / \beta_{\mathrm{V}}^{\prime}$ is the penalty for having to change a train, expressed as an additional in-vehicletime per transfer. Its value is equal to -10 minutes (as used by Dutch Railways (de Keizer et al. (2012))).

- $\beta_{\mathbf{E}}=\beta_{\mathrm{E}}^{\prime} / \beta_{\mathrm{V}}^{\prime}$ is the willingness to arrive to the destination earlier then the desired arrival time, in order to reduce the in-vehicle-time. As shown in Small (1982), the travelers are willing to shift their arrival time by 1 to 2 minutes earlier, if it would save them 1 minute of the in-vehicle-time. We consider here the highest value and set $\beta_{\mathrm{E}}$ to -0.5 .

- $\beta_{\mathrm{L}}=\beta_{\mathrm{L}}^{\prime} / \beta_{\mathrm{V}}^{\prime}$ is the willingness to arrive to the destination later then the desired arrival time, in order to reduce the in-vehicle-time. As shown in Small (1982), the travelers are willing to shift their arrival time by $1 / 3$ to 1 minute later, if it would save them 1 minute of in-vehicle-time. We consider here the highest value and set $\beta_{\mathrm{L}}$ to -1 .

By replacing the original $\beta \mathrm{s}$ in Equation 2 with the estimated ones (i.e. division by $-\beta_{\mathrm{V}}^{\prime}$ ), we obtain function expressed in minutes. This concept is also known as the generalized time $\mathcal{T}$, that is supposed to be minimized by the travelers:

$$
\mathcal{T}_{i t}^{p}=r_{i}^{p}-\beta_{W} \cdot w_{i t}^{p}-\beta_{T} \cdot u^{p}-\beta_{E} \cdot \delta_{i t}^{p}-\beta_{L} \cdot \gamma_{i t}^{p} \quad[\min ]
$$

Since different paths between an origin and a destination may result in different fares, we further monetarize the utility, before we add the fare attribute. The monetarization is done by multiplying the utility by the Value-Of-Time (VOT). The VOT is the willingness-to-pay for travel time savings. The VOT of commuters in Israel (for instance) as of the year 2012 is 21.12 New Israeli Shekel (NIS)/hour (the updated value was given to us by the author of Shiftan et al. (2008)). It basically means that the Israeli travelers are willing to pay in average 21.12 NIS to save one hour of travel time. Converted into the minutes, we get $0.352 \mathrm{NIS} / \mathrm{min}$. By using the VOT, we obtain the generalized cost $\mathcal{C}$ in monetary units and we can simply add the fare $f^{p}$ associated to the itinerary $p$ into its formula: 


$$
\mathcal{C}_{\text {it }}^{\mathrm{p}}=\operatorname{VOT} \cdot \mathcal{T}_{\mathrm{it}}^{\mathrm{p}}+\mathrm{f}^{\mathrm{p}} \quad[\text { monetary }]
$$

Since in the utility theory, the travelers are maximizing their utility and all of the attributes in the generalized cost are making the alternatives less attractive, we need to multiply the Equation 4 by minus one. Indeed the higher the generalized cost is, the lower the value of the utility is. This concept is also known as the passenger satisfaction $\mathcal{S}$ :

$$
\mathcal{S}_{\text {it }}^{\mathrm{p}}=(-1) \cdot \mathcal{C}_{\mathrm{it}}^{\mathrm{p}} \quad[\text { monetary }]
$$

The passenger satisfaction is now the observable part of the utility function $(\mathcal{V}=\mathcal{S})$. Assuming that the unobservable part of the utility function $\epsilon_{i t}^{p}$, is independently and identically distributed according to the Extreme Value distribution, the probability $\operatorname{Pr}_{i t}^{p}$ of a passenger group $(i, t)$ selecting a path $p$ is defined as a logit choice probability:

$$
\operatorname{Pr}_{i t}^{p}\left(w_{i t}^{p}, \delta_{i t}^{p}, \gamma_{i t}^{p}, f^{p} \mid d_{\ell \nu}\right)=\frac{e^{\mu \mathcal{S}_{i t}^{p}}}{\sum_{p^{\prime} \in P_{i t}} e^{\mu \mathcal{S}_{i t}^{p^{\prime}}}}
$$

The probability $\operatorname{Pr}$ is a function of endogenous and exogenous variables. The endogenous ones being the waiting time, the schedule passenger delay and the fare, and the exogenous variable being the timetable (modeled through decisions on departure times $d_{\ell v}$ ). The number of transfers and the in-vehicle-time are constant in the itinerary. They are included inside of the term $\mathcal{S}$. $\mu$ is a scale parameter. This parameter is related to the variance of $\varepsilon$ in Equation 1. It indirectly controls the elasticity of the passenger demand. A last step in calibrating the parameters of the choice probabilities is to find the appropriate value of the scale parameter $\mu$. It can be done by computing the elasticity of the total demand. The aggregate direct point elasticity related to the changes in the generalized cost is given as:

$$
E=\frac{\sum_{i t} \sum_{p \in P_{i t}}\left(\frac{\partial \operatorname{Pr}_{i t}^{p}}{\partial \mathcal{S}_{i t}^{p}} \cdot \frac{\mathcal{S}_{i t}^{p}}{\operatorname{Pr}_{i t}^{p}} \cdot \operatorname{Pr}_{i t}^{p} \cdot n_{i t}\right)}{\sum_{i t} \sum_{p^{\prime} \in P_{i t}} \operatorname{Pr}_{i t}^{p^{\prime}} \cdot n_{i t}}
$$

Upon solving of the derivatives, this transforms into:

$$
E=\frac{\sum_{i t} \sum_{p \in P_{i t}}\left(1-\operatorname{Pr}_{i t}^{p}\right) \cdot \mu \cdot \operatorname{Pr}_{i t}^{p} \cdot n_{i t}}{\sum_{i t} \sum_{p^{\prime} \in P_{i t}} \operatorname{Pr}_{i t}^{p^{\prime}} \cdot n_{i t}}
$$

According to Whelan and Johnson (2004), the value of the elasticity for the railway passenger demand with respect to the fare and the generalized cost is $E=-0.58$. Therefore, the value of $\mu$ can be calibrated by solving Equation 8 for any case study. In our application, we have calibrated the value of $\mu$ to 0.06 .

Remark In this problem, we use probabilistic passenger assignment based on a Multinomial Logit (MNL) model. MNL holds IIA condition (Indepence of Irrelevant Alternatives). This property is a well known and well documented limitation of such models in route choice problems due to the 
physical overlap of the paths (Ben-Akiva and Bierlaire (2003)). In this paper, as our focus is on the methodological integration, a Multinomial logit model has been deemed appropriate for the proof-ofconcept. In real case study, with real demand data (if they available), more advanced models (e.g. path-size logit) could be used to correct the issue.

\section{Problem Definition}

In this section, we present the Elastic Passenger Centric Train Timetabling Problem (EPCTTP) which is an extension of the PCTTP model defined by Robenek et al. (2016). They showed that the major improvement of this framework is in the networks with high density of the passengers. These networks generally operate with double-track railways. Moreover, our interest is more on studying the problem for regional or inter-city rails. The presented approach can also be applied to other railway services as long as it satisfies the underlying assumptions.

The aim of the EPCTTP is to design a desirable timetable maximizing the TOC's revenue accounting for the passenger forecasts (probabilistic demand). Whereas the aim of the original PCTTP was to design a desirable timetable maximizing the overall passenger satisfaction (without the fare), thus securing the social optimum (the actual choice of the passengers were unknown, i.e. the demand was deterministic). Moreover, the passenger assignment in the PCTTP was from the central planner's point-of-view, whereas the EPCTTP combines the First Come First Serve (FCFS) policy with the revenue maximization (in the case of a tie). We compare the performance of the two models in Section 6.3. Here we should note that in desirable timetable, one is interested to know the most suitable depature time of the trains. The desirable timetable can then be adjusted in later planning stage to assure track capacity which determines the "actual" timetable. Interested readers are referred to Caprara et al. (2002) to determine the actual timetable based on the desirable one. Therefore, in this paper, we assume that running time and dwell times are fixed. This way we allow higher level of flexibility to the approach used in the later stage of timetabling.

This section provides only the formal definition of the problem. For the reader's convenience, we organize all the mathematical notation in Table 6 in Appendix C. The mathematical formulation of the EPCTTP can be found in Appendix $\mathrm{C}$ as well.

A train is defined by its line $\ell \in \mathrm{L}$, i.e. the set of stations that it serves. Each line $\ell$ has a train frequency and therefore, a specific train is given by a combination of indices $(\ell, v)$, where $v \in \mathrm{V}^{\ell}$ is the train's position within the set of available trains of the line $\ell$. Note, that in some literature, the term train refers to the physical rolling stock. Therefore, the term service might be used instead for the set $v \in \mathrm{V}^{\ell}$. Both lines and frequencies are given by the Line Planning Problem (LPP). Moreover, we assume that the line frequencies obtained from line planning problem are feasible (i.e. at least one actual timetable can be constructed respecting line frequencies)

In this framework a line is uni-directional, i.e trains in the other direction are serving a conceptually different line A timetable is defined as a set of arrival and departure times of each train $(\ell, v)$. Since the travel times $r_{i}^{p}$ consisting in dwell times and running times in between stations are deterministic, it is sufficient to decide only on the departure time $d_{\ell v}$ of each train $(\ell, v)$ from its origin station. The problem can design three types of timetable: non-cyclic (by default), cyclic (imposing 
cyclicity constraints) and hybrid cyclic (imposing hybrid cyclicity constraints).

Based on the set of trains, the set of paths $P_{i}$ for each OD pair $i$ is given. Each passenger group is having its own set of available paths based on the original one: $P_{i t} \subseteq P_{i}$. Any path can consist in several consecutive trains (limited to 3 in this study), in the order in which they are used, i.e. a passenger takes the first train and then transfers to its second train, etc. If a transfer is not possible (destination train departs before the arrival of the origin train), the path is removed from the set $P_{i t}$. The set of all time-independent paths is pre-processed and can be created using a method which is described in Appendix A. When making a transfer from one train to another, a minimum transfer time $\mathrm{m}$ is always secured. Any additional time spent in the transferring stations is counted as a waiting time. The set of paths contains the opt-out option which is equal to the shortest path, where the passengers do not experience any waiting time and arrive to their destinations exactly on their desired arrival time $a_{i t}$.

Note that the problem is designed to be ran by a single TOC to optimize his/her timetables. However, in case that a competing operator exists, his/her schedule can be included in the set of paths. In such a case, the departure times need to be fixed to its values as well as the ticket prices. The revenue generated by the competitor's service needs to be set to zero.

The passengers are assigned to trains through the demand forecasting model presented in the previous section. The probability of a passenger group $(i, t)$ selecting a path $p$ is given by Equation 6 . The groups of passengers can be split according to the probabilities of each path. Since the passengers can enter or leave a train at any of its stopping stations, each train is further decomposed into segments $s \in S^{\ell}$. The occupation $o_{\ell v}^{s}$ of any train $(\ell, v)$ on any of its segments $s$ cannot exceed its capacity $W$. The problem assumes a homogenous fleet of trains, but it can be easily adapted to a heterogenous one. If a train reaches a capacity on some of its segments, all the paths containing this segment of this train of leftover passengers are removed from their respective choice sets. The passengers are assigned to trains based on their probabilities and the FCFS policy. If there is a tie among the passengers, then the ones who bring more revenue are assigned on the train (in line with the objective function of revenue maximization).

The revenue of a TOC is calculated as a function of the collected fares $f_{\ell v}^{s}$ for each train on each of its segments (multiplied by the train occupation). The fare itself is a decision variable and serves as an instrument to either flatten the demand, increase the revenue or to attract further passengers. The total fare $f^{p}$ is a sum of all fares on all segments of all trains $f_{\ell v}^{s}$. In practice, the operators use various fare systems. Equation 4 represents the generalized cost (within the utility function) associated with each passenger and their choices. As this function is in monetary units and all information about the journey is relayed through the path and the passenger, it can be used for any fare structure, as long as it can be expressed as the fare structure associated to each path.

In this problem, we assume that the train operating company has to run a given number of identical trains (obtained by frequency setting in line planning problem). In addition, the average operating cost of the train (per km per day) is also given. Therefore, the operating cost associated to each timetable is a constant value in our model and can be removed from the objective function. In other words, by maximizing the revenue one can determine the maximum profit.

The above model would produce a non-cyclic timetable. However, by adding some additional constraints, the model can produce any type of a timetable. In this manuscript, we consider two 
additional timetable types: cyclic and hybrid cyclic.

Cyclic Timetable The cyclicity originates from the Periodic Event Scheduling Problem (PESP) first defined by Serafini and Ukovich (1989). The PESP secures that a set of events is scheduled in equally spaced intervals. In the railway context, the departure times of the trains on the same line are scheduled in cycles of size $c$. Typical value of $c$ is 60 minutes leading to a clock-faced timetable. Such a measure leads to a better memorability of a timetable by the passengers. To obtain a cyclic timetable, the below constraints need to be included in the EPCTTP:

$$
\begin{array}{cl}
\mathrm{d}_{\ell v}-\mathrm{d}_{\ell v-1}=\mathrm{c} \cdot z_{\ell v}, & \forall \ell \in \mathrm{L}, \forall v \in \mathrm{V}^{\ell}: v>1, \\
z_{\ell v} \in \mathbb{N}^{+}, & \forall \ell \in \mathrm{L}, \forall v \in \mathrm{V}^{\ell} .
\end{array}
$$

Constraints (9) secure that the departure time of two consecutive trains $v$ and $v-1$ of each line $\ell$ are spaced in the multiples $z_{\ell v}$ of cycles $c$. The domain constraints (10) verify that these multiples are non-negative natural numbers.

Hybrid Cyclic Timetable The cyclic timetable, when compared to other types of timetable, might be too restrictive and in fact it may lead to worse solutions in terms of the passenger satisfaction. Instead of using a fully cyclic timetable, Robenek et al. (2017) propose a novel hybrid cyclic timetable combining the benefits of both cyclic and non-cyclic timetables. They show that the novel timetable can achieve similar performance as the non-cyclic one in terms of the passenger satisfaction.

Overall, the aim of the hybrid cyclic timetable is to decide on the ratio between the cyclic and the non-cyclic trains. It discretizes the planning horizon $H$ into a set of unique cycles $k \in K: K=H / c$. The cyclic and non-cyclic trains have to follow the below rules for each cycle $k$ per each line:

- Any cycle $k$ can contain no train

- Any cycle $k$ can contain at maximum one cyclic train

- Any cycle $k$ can contain one or more non-cyclic trains, only if there is a cyclic train present inside the kth cycle

From the above rules, we define additional binary decision variables: $\mathrm{q}_{\ell v}$ and $\mathrm{y}_{\mathrm{k}}^{\ell}$. $\mathrm{q}$ variable indicates if a train $(\ell, v)$ is a cyclic one $(=1)$ or a non-cyclic one $(=0)$. $y$ variable indicates if a line $\ell$ is having a cyclic train scheduled in the cycle $k(=1), 0$ otherwise.

\section{Solution Algorithm}

The EPCTTP formulation has non-linear constrains resulted from using the discrete choice model. In literature, the non-linear logit model can be linearly approximated by using piece-wise linear constraints (Marín and García-Ródenas (2009)). However, due to the complexity involved in designing 
the timetable and our wish to solve the problem for large instances, we propose a heuristic algorithm which directly handles non-linear constraints. Given that the original formulation of the PCTTP has been solved successfully with a Simulated Annealing (SA) heuristic by Robenek et al. (2017), we use the same algorithm with some changes to reflect the specific features of the EPCTTP formulation. The changes are related to the value function (revenue maximization based on probabilities instead of deterministic passenger satisfaction maximization), the passenger assignment (user optimum to reflect better passenger behavior instead of system optimum) and new neighborhood moves (for the pricing of the tickets). Even though the heuristic has been validated as compared to CPLEX in Robenek et al. (2017), its performance is not always guaranteed. In this manuscript, the results of the heuristic are later compared to a benchmark of a real case study.

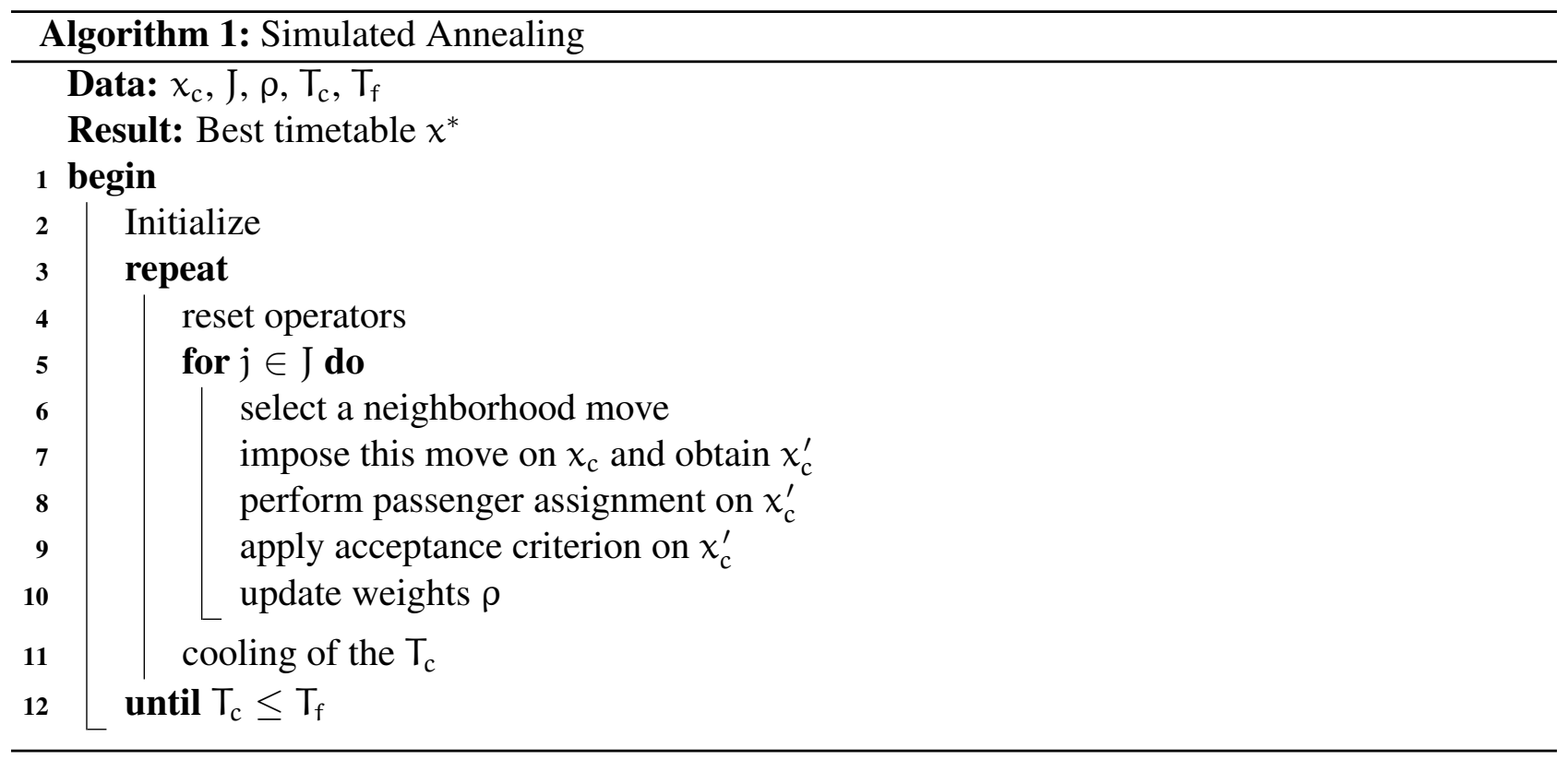

The Algorithm 1 shows the general pseudo-code of the heuristic. The SA heuristic was first defined by Kirkpatrick et al. (1983). The algorithm takes as an input an initial solution $x_{0}$ that consists in the departure times of each train, and the initial fares per line and per segment. Since we solve this heuristic for several types of timetables (cyclic, hybrid cyclic and non-cyclic), an initial feasible timetable for any type of these timetables is any cyclic timetable. In our case study, we use the currently operated (cyclic) timetable of Israeli Railways. Similarly for the fares: we use the current prices of Israeli Railways. If fares are not available, one can assign ad-hoc values proportional to the length of each segment. The heuristic starts off with an initial temperature $T_{0}$ that has been previously calibrated to be a function of the value of the initial solution $f\left(x_{0}\right)$ as follows: $T_{0}=10^{-5} \cdot f\left(x_{0}\right)$. This temperature is gradually cooled down up until the final temperature $T_{f}$ is reached. The cooling scheme has been calibrated to be $5 \%$ of the initial temperature $T_{0}$ and a standard value of the final temperature $\mathrm{T}_{\mathrm{f}}$ of 0 has been previously used.

For each temperature, the algorithm performs $J=1000$ iterations (previous calibration). In each iteration $j$, the current solution $x_{c}$ is adjusted with one of the several neighborhood moves. Two main 
categories of neighborhoods exist: fare adjusting and timetable adjusting.

The fare adjusting neighborhood consists in a single move: a train $(\ell, v)$ is randomly selected through subsequent uniform distributions $\mathcal{U}(1,|\mathrm{~L}|), \mathcal{U}\left(1,\left|\mathrm{~V}^{\ell}\right|\right)$. Since fare is related to a segment, this move also selects a random segment crossed by the chosen train according to $\mathcal{U}\left(1,\left|S^{\ell}\right|\right)$. The fare of this segment can be either increased or decreased based on a uniform probability of 50:50\%. The actual fare is then adjusted by a value between 1 and 5 monetary units decided by the uniform distribution $\mathcal{U}(1,5)$.

The timetable adjusting neighborhood(s) consists in several moves dependent on the type of the timetable solved. The overview of the timetable specific moves can be found in Table 1 . The columns represent the moves per timetable type and the rows represent a stage of a move. Only the cells with a grey background constitute a move. The last column represents the underlying distributions from which to draw randomly, in order to obtain the respective attribute's value.

\begin{tabular}{|c|c|c|c|c|c|}
\hline & & Non-C. & Cyclic & Hybrid Cyclic & Distribution \\
\hline \multirow{4}{*}{ Select } & $(\ell, v)$ & & & & $\mathcal{U}(1,|\mathrm{~L}|), \mathcal{U}\left(1,\left|\mathcal{V}^{\ell}\right|\right)$ \\
\hline & $\ell$ & & & & $\mathcal{U}(1,|\mathrm{~L}|)$ \\
\hline & $\mathrm{q}_{\ell v}=0$ & & & & \\
\hline & $\mathrm{q}_{\ell v}=1$ & & & & \\
\hline \multirow{3}{*}{ Modify } & $\mathrm{d}_{\ell v}$ & & & & $\mathcal{U}(0, \mathrm{H}-1)$ \\
\hline & $\mathrm{d}_{\ell v} \bmod \mathrm{c}$ & & & & $\mathcal{U}(0, c-1)$ \\
\hline & k & & & & $\mathcal{U}(0, \mathrm{H} / \mathrm{c}-1)$ \\
\hline \multirow{3}{*}{ Apply } & $\forall v \in \mathrm{V}^{\ell}: \mathrm{q}_{\ell v}=1$ & & & & \\
\hline & $y_{k}^{\ell}=0$ & & & & \\
\hline & $y_{d_{\ell v} / c}^{\ell}=1$ & & & & \\
\hline
\end{tabular}

Table 1: Overview of the neighborhood moves by the type of a timetable

A timetable move is imposed on the current solution $x_{c}$ in 3 steps: selection, modification and application. The selection step serves to identify that part of the current solution $x_{c}$ that is to be modified. The modification step serves to identify new value(s) for the selected part of the solution. The application step effectuates the change.

In the selection stage, two entities can be selected: a specific train $(\ell, v)$ or the whole line $\ell$. The selection of a specific train might be conditioned by its type $\left(\mathrm{q}_{\ell v}=1\right.$ means a cyclic train needs to be selected, $\mathrm{q}_{\ell v}=0$ means a non-cyclic train needs to be selected). If no condition is specified, any train can be selected. When selecting a whole line, no conditioning is needed.

The aim of the modification is to find a new value for one of the 3 attributes. The concerned attributes are: the departure time $d_{\ell v}$, the modulo remainder $d_{\ell v} \bmod c$ and the kth cycle of a train. The modulo attribute represents the modulo time within any cycle. For instance, when the cycle $c$ is equal to one hour and the departure time of a train is 5:45, then the modulo time is 45 minutes. This time is the same among all cyclic trains of the same line. The kth cycle is the cycle in which the train is departing. For the previous example of a train departing at 5:45, the $k=5$. 
The application of the modification is always performed on the pre-selected entity. However, its application might be conditioned. Some applications are made only to the cyclic trains of the given line $\left(\forall q_{\ell v}=1\right)$. Other applications are conditioned that the new cycle $k$ does not already contain a cyclic train $\left(y_{k}^{\ell}=0\right)$ or that the new departure time $d_{\ell v}$ is in a cycle that does already contain a cyclic train $\left(y_{d_{\ell v} / c}^{\ell}=1\right)$. If the newly generated values of attributes fail to fulfill the last two conditions (when they are required), the modification is repeated (until they do).

For illustration, consider the first move of the hybrid cyclic timetable: at first a train is selected by drawing the line $\ell$ from $\mathcal{U}(1,|\mathrm{~L}|)$ and subsequently drawing the $v$ from $\mathcal{U}\left(1,\left|\mathrm{~V}^{\ell}\right|\right)$. The drawing from the distribution(s) is repeated until the condition $\mathrm{q}_{\ell v}=0$ is satisfied. Hence, a non-cyclic train is selected. In the second phase, a new departure time $d_{\ell v}$ of this train is drawn from $\mathcal{U}(0, \mathrm{H}-1)$. In the third phase, this new departure time is applied given that it is in the cycle where there is a cyclic train scheduled $\left(y_{d_{\ell v} / c}^{\ell}=1\right)$. Otherwise the modification phase is repeated until this condition is respected.

Each neighborhood move is assigned a weight $\rho$ that is initialized to 1 at each new temperature (in order to avoid one operator dominating the others). In each iteration, a neighborhood move is selected using a roulette wheel mechanism based on the current weights $\rho$ of all moves. The values of $\rho$ s are updated based on their performance with respect to the acceptance criteria of the new solution $x_{c^{\prime}}$ :

- If $f\left(x_{c^{\prime}}\right) \geq f\left(x^{*}\right) \rightarrow \rho=\rho+3$

- If $f\left(x_{c^{\prime}}\right) \geq f\left(x_{c}\right) \rightarrow \rho=\rho+2$

- If $f\left(x_{c^{\prime}}\right)<f\left(x_{c}\right)$ and the new solution is accepted with a probability $r<\exp \left(-\left(x_{c}-x_{c^{\prime}}\right) / T_{c}\right)$, where $r$ is drawn from $\mathcal{U}(0,1)$, then $\rho=\rho+1$

Once a new solution $x_{c}^{\prime}$ is obtained (by applying a selected move), its revenue is calculated using the passenger assignment in Algorithm 2. Note, that the exhaustive list of routes (i.e. $\hat{P}_{i}$ has been generated only once in the beginning of the heuristic as described in Appendix A. This set contains also the opt-out option. During the timetable adjustment phase, the set of active paths are determines $P_{i t}$. The passenger assignment procedure only assigns probabilities to the active paths. Note that the opt-out option is always active. In order to model the passenger behavior as realistically as possible, the assignment is using a First Come First Serve (FCFS) policy. At the beginning, the probabilities of all paths of all passengers are predicted. The passengers are then assigned to the trains in their paths with their probabilities in a non-capacitated fashion. Afterwards, the algorithm enters a while loop that serves to resolve any capacity issues. It iterates through all overflowing trains (i.e. those exceeding their capacity) that have been sorted by their descending occupation. If a train exceeds its capacity on any of its segments, the passengers that have entered this train on this specific segment are sorted ascending according to the overall revenue that they bring to the company (the FCFS policy i.e. only the entering passengers can be used to resolve the capacity issues, the ones that are already on the train cannot be asked to leave the train). Even though the passengers do not behave this way (they would sort themselves randomly), this policy is in line with the objective of revenue maximization. Afterwards, the algorithm removes these passengers one by one until the occupation of this train on this segment does not exceed its capacity. For each of the removed passengers, the path that they were using to travel on the segment of the concerned train is removed from their choice set and 


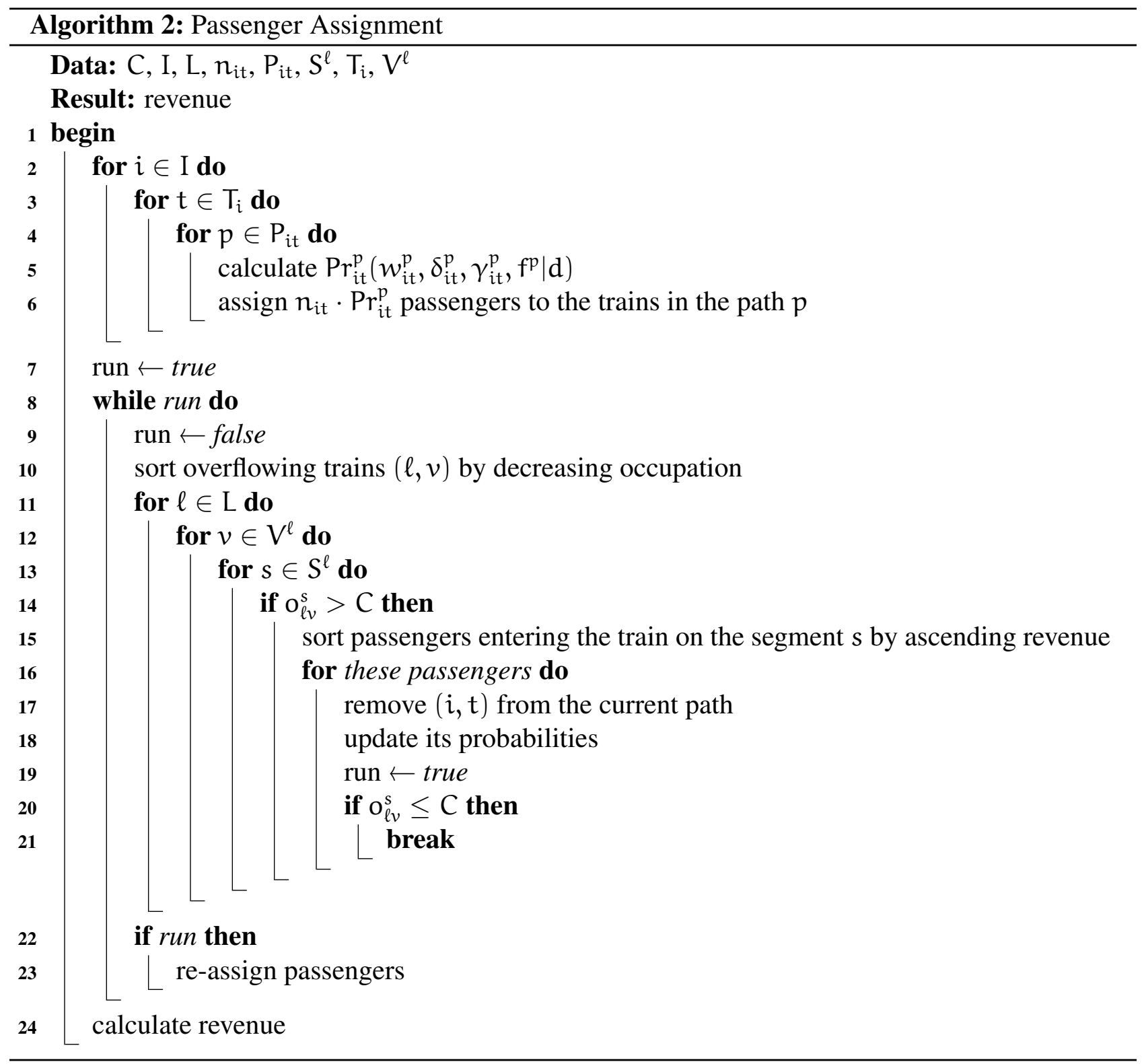


the probabilities of their remaining paths are recalculated. Once all the conflicts (of this iteration of the while loop) are resolved, the algorithm again re-assigns all the passengers to the trains in a non-capacitated fashion from scratch (the new probabilities of the removed passengers affect other passengers and trains). The algorithm terminates when there are no capacity issues found in a single iteration of the while loop. Lastly, the algorithm calculates the overall revenue of the current solution $x_{c^{\prime}}$.

The heuristic terminates, when the final temperature $T_{f}$ is reached.

\section{Case Study}

As our case study, we have selected the network of Israeli Railways (in Figure 2). The aim is to evaluate the effect of different timetables on the revenue and what is the potential increase in the revenue, when the timetable design is integrated with ticket pricing. The exact procedures, assumptions and information about the data can be found in Appendix A.

We consider two instances in our study: the 2008 situation and the 2014 situation. The 2008 situation is built from the ticket selling machines' data of an average working day (from 6 a.m. to 1 a.m.) in 2008 in Israel. This data was kindly provided to us by Mor Kaspi and Tal Raviv, who have used it in their study Kaspi and Raviv (2013). In 2008, there are 126036 passengers. The 2014 scenarios assume that the structure of the demand is the same as in 2008, with a uniform inflation of 1.6, resulting in a total number of 193886 passengers (based on the newspaper article in Globes (2015)).

We consider the network layout of 2008: there were 47 stations and the red line in Figure 2 was operated only between Hod HaSharon and Tel Aviv - HaHagana. We do not consider the night line (blue with black bordering) as it runs mainly in the period for which we do not have the demand data (i.e. between 1 a.m. and 6 a.m.). Even though there are only 18 unidirectional lines visualized in Figure 2, in reality there are 34 unidirectional lines in the timetable (some lines are operated with higher frequency of 2 or more cyclic times, e.g. every xx:15 and xx:45). Since some of the trains follow different stopping patterns within a line, we have taken a union of stopping stations for each line (but same colored lines with different cyclic times may operate different stopping patterns). Since the OD matrix is given for an average working day, we have removed the trains that operate only during the holidays.

The timetable operated in Israel is cyclic with a cycle of 60 minutes. We use the timetable of 2013/14 (Unlike Europe, the timetable change in Israel happens during the summer period, i.e. the naming 13/14), which was the latest published timetable at the time of the implementation, where 6 out of 388 trains have non-cyclic departure times. The planning horizon $\mathrm{H}$ of this case study is one day. Even though the demand is between 6 a.m. and 1 a.m., we allow the PCTTP model to schedule trains during any time of the day. We solve the PCTTP using the SA heuristic for the following timetables:

- IR 13/14 - the departure times are fixed to the ones of IR timetable of 13/14. Since the departure times are fixed, it serves as the benchmark for comparison of the performance of the other timetables. 


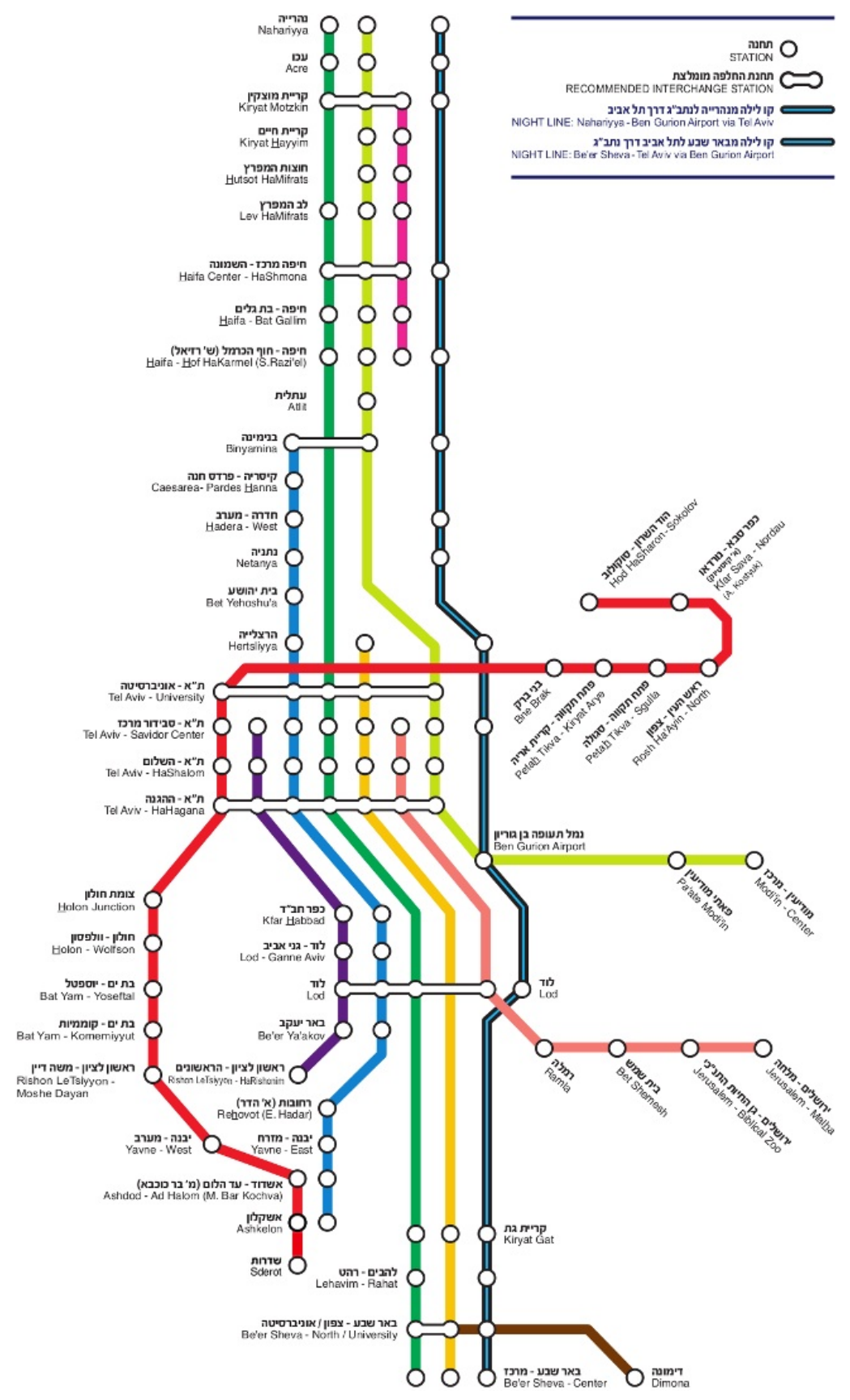

Figure 2: Network of Israeli Railways (www.rail.co.il)

- Non-Cyclic - no specific rule on the departure times is enforced.

- Cyclic - the departure times have to be cyclic according to constraints (9) - (10).

- Hybrid Cyclic - the departure times have to comply with hybrid cyclicity conditions described in Section 4. 
The revenues of each type of timetable are given in NIS. At first, the problem is solved without pricing and subsequently, it is solved with pricing. Each time, the heuristic is given as an initial solution the previously found timetables (IR timetables for the cyclic version, cyclic for the hybrid cyclic version and the hybrid cyclic for the non-cyclic one). The solution methodology has been coded in Java and it was ran on a Unix server with up to 24 cores of $3.33 \mathrm{GHz}$ and $62 \mathrm{GiB}$ RAM.

\subsection{Results without pricing}

The results of the EPCTTP model without pricing can be found in Tables 2 and 3. Therefore, the prices in the model are fixed to those used by Israeli Railways (obtained from IR's website www.rail.co.il). The columns represent the different types of timetables (as described above) and the rows show the values of various attributes. The concerned attributes are: the revenue, the revenue gain as compared to the initial IR 13/14 timetable, the market share (percentage of passengers transported by IR), the number of passengers transported by IR, the average and the median train occupation and the solution time of the heuristic.

\begin{tabular}{r|rrrrr}
\hline & IR 13/14 & cyclic & hybrid cyclic & non-cyclic \\
\hline revenue [NIS] & 4865777 & 5083828 & 5299618 & 5269661 \\
revenue gain [NIS] & - & +218051 & +433841 & +403884 \\
market share [\%] & 73.61 & 75.47 & 77.96 & - \\
\# transported passengers & 92779 & 95127 & 98267 & - \\
avg. train occupation & 116 & 121 & 126 & - \\
median train occupation & 84 & 90 & 98 & - \\
solution time [sec] & 7 & 174897 & 164828 & 167939 \\
\hline
\end{tabular}

Table 2: Computational results of the various timetables for 2008 situation without pricing

\begin{tabular}{r|rrrrr}
\hline & IR 13/14 & cyclic & hybrid cyclic & non-cyclic \\
\hline revenue [NIS] & 7150254 & 7490054 & 7896806 & 7905615 \\
revenue gain [NIS] & - & +339800 & +746552 & +755361 \\
market share [\%] & 70.63 & 72.78 & 75.56 & 75.59 \\
\# transported passengers & 136981 & 141143 & 146535 & 146606 \\
avg. train occupation & 172 & 180 & 189 & 189 \\
median train occupation & 130 & 141 & 159 & 157 \\
solution time [sec] & 11 & 287531 & 295501 & 298114 \\
\hline
\end{tabular}

Table 3: Computational results of the various timetables for 2014 situation without pricing

In all cases, the SA algorithm terminates after 300,000 seconds. The reason is that we set 1000 iterations for each temperature unit. Moreover, the solution time also depends on the number of passengers' groups. 
Both tables show that taking into account passenger behavior leads to better performing timetables (in all of the concerned attributes). The EPCTTP based timetables were able to attract back some passengers from the competition and thus increase the IR's market share. The average revenue increase is $5 \%$ and $10 \%$ for the cyclic and hybrid cyclic timetable respectively.

We used the hybrid cyclic timetable as the initial solution for the non-cyclic one in the 2008 situation. However, it has failed to find a better solution than the hybrid cyclic one. In the 2014 situation, it was able to improve the solution only marginally. The marginal differences are caused by the randomness of the heuristic approach. The similarity, in the solutions of the two types of timetables, shows that the concept of the hybrid cyclic timetable can diminish any impact of the cyclicity constraints on the performance of the timetable (the impact being the differences between a cyclic and a non-cyclic solution).

The average ratio between the cyclic and the non-cyclic trains, in the hybrid cyclic timetable, is around 50:50\%. The solution time of the heuristics was on average 2 (for 2008) and 3 (for 2014) days.

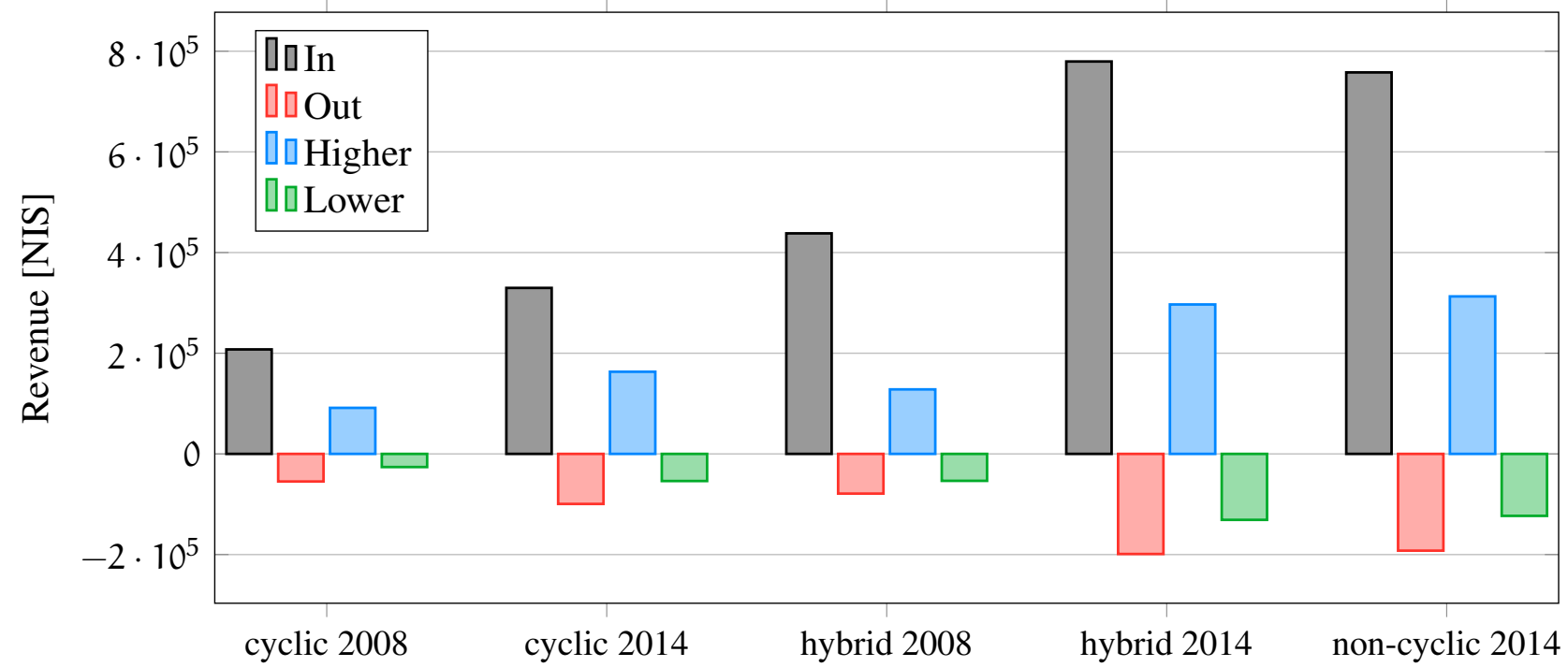

Figure 3: Difference in composition of passengers for the various timetables

In order to better understand, what is the cause of the additional revenue, we plot the difference in composition of passengers per timetable as compared to the IR 13/14 timetable in Figure 3 . This Figure shows the revenues generated by 4 different groups of passengers: in - the ones who were not using IR in the original timetable, out - the ones who were using IR in the original timetable and left, higher - the passengers who stay and pay more, lower - the passengers who stay and pay less. The sum of these groups per timetable provides the value of revenue gain. It can be seen that the main source of the additional revenue across timetables is the new coming passengers. However, we can see that in the hybrid cyclic and non-cyclic timetables for the situation in 2014, the strategy also includes preference to the more paying passengers. The cause of this is the fact that in the case of a tie the passengers who pay larger fare (i.e. longer distance passengers) gain preference. Therefore, some of the shorter distance passengers might choose a path that is longer than their shortest path and some of them might opt-out (if the marginal utility loss to the opt-out option is too high). 


\subsection{Results with pricing}

The results of the EPCTTP model with pricing can be found in Table 4. The prices are now decision variables with their initial values set to those of IR. The revenue gain is now the additional revenue as compared to the solution of the same timetable without pricing. The overall revenue shows the additional profit as compared to the original IR 13/14 timetable.

\begin{tabular}{|c|c|c|c|c|c|c|}
\hline & \multicolumn{2}{|c|}{ cyclic } & \multicolumn{2}{|c|}{ hybrid cyclic } & \multicolumn{2}{|c|}{ non-cyclic } \\
\hline & 2008 & 2014 & 2008 & 2014 & 2008 & 2014 \\
\hline revenue [NIS] & 5256959 & 7767140 & 5461559 & 8229227 & - & 8259557 \\
\hline revenue gain [NIS] & +173132 & +277087 & +161942 & +332421 & - & +353942 \\
\hline market share [\%] & 76.31 & 74.05 & 76.87 & 75.99 & - & 76.32 \\
\hline \# transported passengers & 93848 & 139451 & 96886 & 147373 & - & 148017 \\
\hline avg. train occu & 127 & 188 & 128 & 194 & - & 196 \\
\hline median train occupation & 95 & 148 & 99 & 156 & - & 157 \\
\hline solution time [sec] & 97105 & 185560 & 89044 & 197695 & - & 182810 \\
\hline overall revenue gain [NIS] & +391183 & +616887 & +595783 & +1078973 & - & +1 109303 \\
\hline
\end{tabular}

Table 4: Computational results of the various timetables with pricing

The pricing was able to further improve the revenue of the timetables. All timetables increased the ridership with exception of the hybrid cyclic one for the 2008 situation. However, it has still managed to achieve a higher profit. This is due to the fact, that the passengers who left were generating lesser profit than the ones who came in (Figure 4(e)). The additional increase in the revenues by pricing is up to $5 \%$. We used the hybrid cyclic timetable as the initial solution for the non-cyclic one in the 2008 situation. However, it has failed to find a better solution than the hybrid cyclic one.

In order to analyze the pricing scheme, we plot various graphs in Figure 4. At first, we look at the pricing variations by location - Figures 4(a) and 4(b). Both plots show the overall increase/decrease in price per segment of the network as compared to the original prices set by Israeli Railways. The $x$ axis represents the segments by volumes of passengers traveling on them. For low demand segments $(<20000$ pax) the pricing can go either way, whereas for the high demand segments ( $>20000$ pax) the prices go strictly up. The boundary between the two pricing strategies is around 20000 passengers per segment.

Subsequently, we look at the price variations based on the time of the day - Figures 4(c) and 4(d). Both plots show the overall increase/decrease in price per hour in the network as compared to the original prices set by Israeli Railways. The figures exhibit clear patterns on how to adjust the pricing: increase the price for high demand density periods and decrease the price for low demand density periods. Since the morning peak is more dense, its prices are higher than of the evening peak (the evening demand is more spread). The price decreases, as compared to the increase in peak hours, is lower. This might be due to the fact that during the off-peak hours also the supply is more scarce, i.e. the prices can be higher. The cyclic timetable has less extreme changes than the hybrid cyclic one. This is due to the fact that the cyclicity makes the trains more spread. Therefore, the results in 


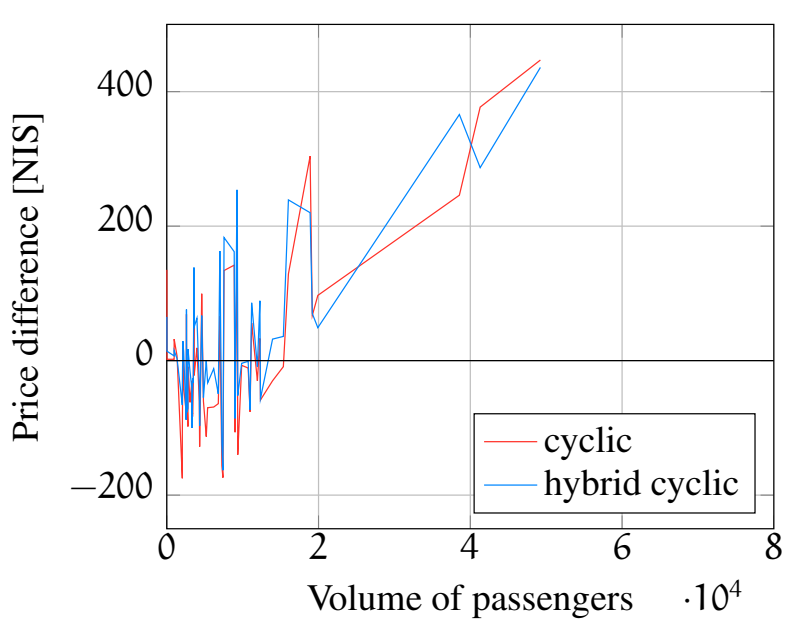

(a) By location - 2008

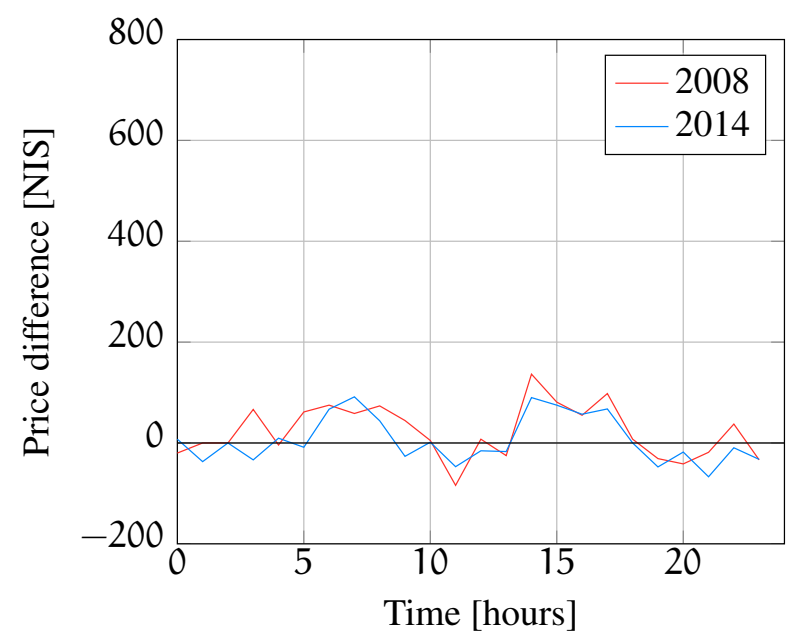

(c) By time - Cyclic

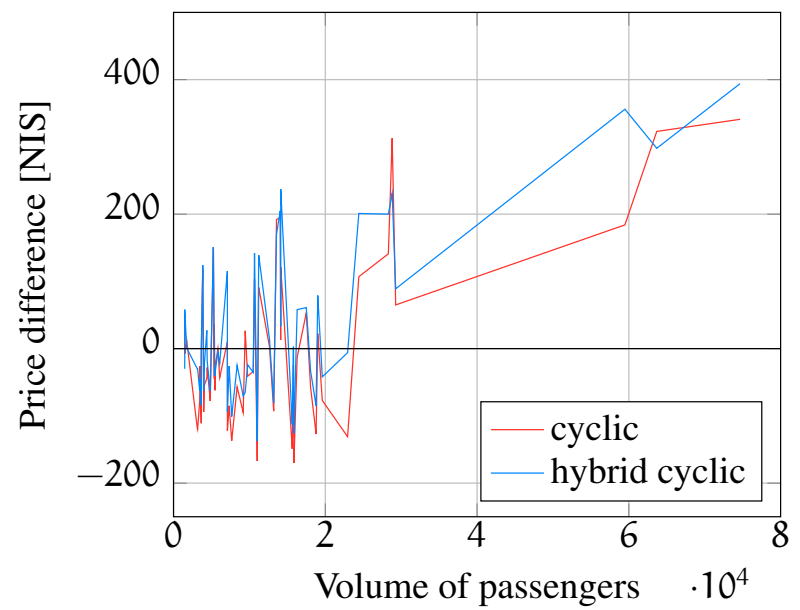

(b) By location - 2014

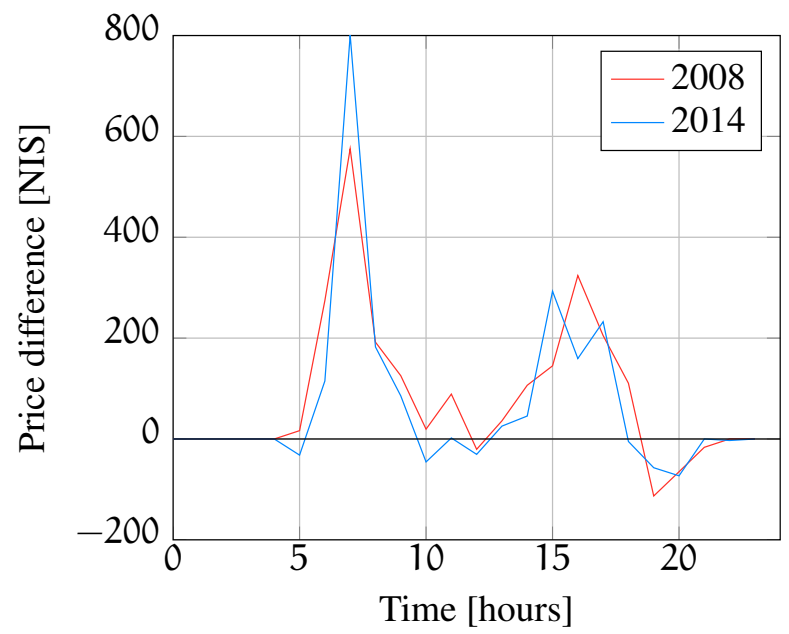

(d) By time - Hybrid cyclic

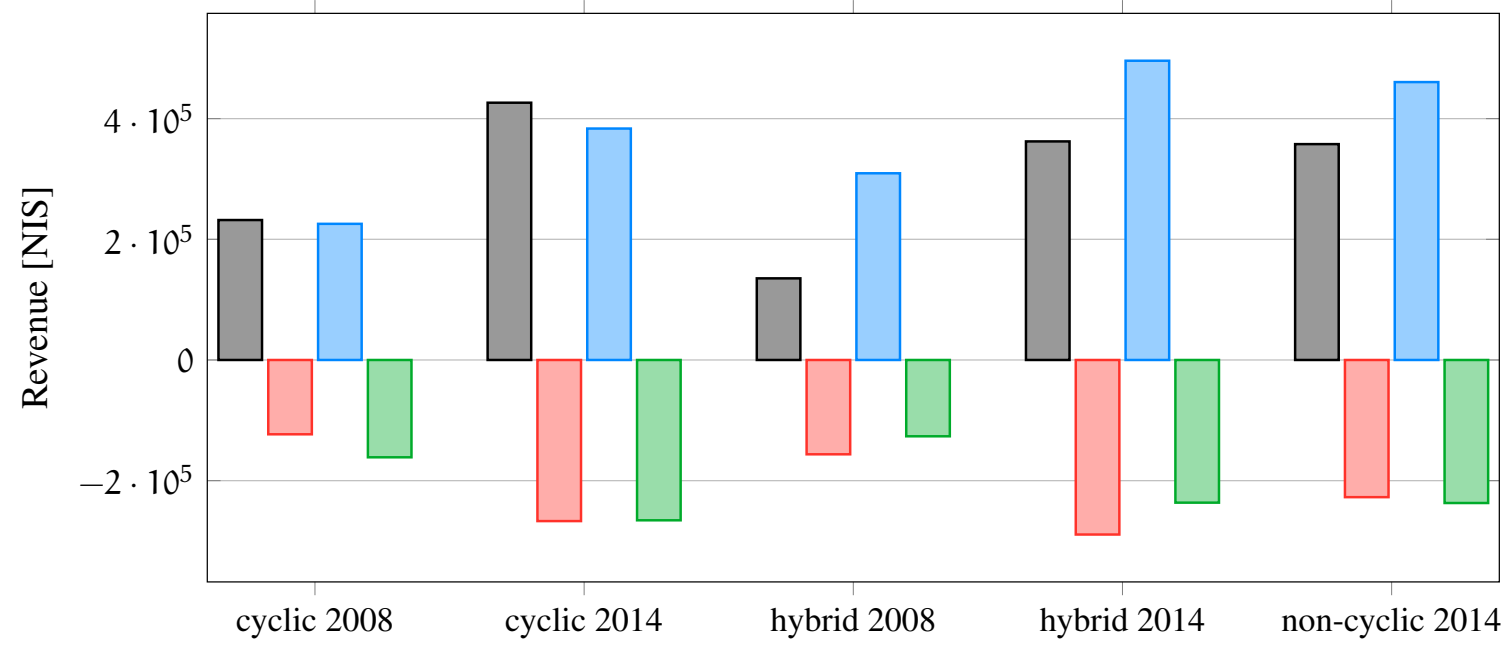

(e) Difference in composition of passengers for the various timetables (same legend as in Figure 3)

Figure 4: Pricing analysis 
Figure 4(c) are somewhat more aggregate (the hours contain a larger mix of trains with increased and decreased prices).

Finally, to understand the changes in the served passengers, we plot the differences in the composition of the passengers for various timetables as compared to their previous versions without pricing in Figure 4(e). The legend is the same as in Figure 3. It can be seen, that as compared to the timetabling without pricing, the higher paying passengers are now having a larger contribution to the revenue gain. In the hybrid and non-cyclic timetables, the share is even larger than that of the new coming passengers. The revenue loss of leaving and less paying passengers is now larger. These passengers are most likely left out or less paying, in order to free the space in the trains desired by the higher paying passengers. This behavior is in line with the objective of maximizing revenue, but might not be desired by public owned operators. Therefore, we further investigate what is the impact of this approach on the passengers' satisfaction, in the next Section.

\subsection{Maximize satisfaction or maximize revenue?}

Maximizing revenues is a natural objective in a competitive environment. However, in many countries, particularly in Europe, there is little or no competition. Moreover, many railway operators are subsidized by public money, and must focus on public services as well. Therefore, the revenue maximization might be considered controversial. In order to analyze the situation, two approaches are evaluated against each other: one timetable designed to maximize the satisfaction of the passengers (Robenek et al. (2017)), and one timetable designed to maximize revenues, as described in this paper. In order to do so, the timetables (i.e. the decision variables $d_{\ell v}$ ) created by Robenek et al. (2017) (through the passenger satisfaction maximization) are given as a fixed input to the probabilistic model of EPCTTP. The comparison consists in the resulting revenue, satisfaction and number of transported passengers per timetable in Table 5.

In this Table, $(s)$ denotes satisfaction maximization approach, $(r)$ denotes the revenue maximization approach and $(p)$ denotes the presence of prices generated by the pricing problem. If $(p)$ is missing, the original prices given by IR are used. Note that in the 2008 situation, the hybrid cyclic timetable was not designed using the satisfaction approach (due to the insignificant differences in satisfaction among the cyclic and non-cyclic timetables in 2008 (Robenek et al. (2017))). The noncyclic timetable in 2008 with pricing is not included as it did not improve the revenue and its fares were never reported.

The main observation from the results is that even though the objectives were different, the resulting passenger satisfaction is more or less similar. Indeed, when maximizing the revenue, the passengers need to be satisfied, in order to take the train. The main difference, between the two approaches, is the generated revenue. This is again in line with the objectives: the objective of revenue maximization takes care of both revenue and satisfaction, whereas the other approach only cares about the satisfaction. Notable to mention, that the presence of pricing did not distort the average values of the satisfaction. Its values are similar with and without pricing. The cause of it is most likely the reduced fare for some of the passengers.

It could be objected that the satisfaction is now similar across conceptually different timetables and that it is caused by the opt-out option of the competitor. However, given that the number of 


\begin{tabular}{r|rrr}
\hline & revenue [NIS] & satisfaction [NIS] & \# transported passengers \\
\hline cyclic 2008 (s) & 4950614 & -10264108 & 93580 \\
cyclic 2008 (r) & 5083827 & -10314244 & 95127 \\
\hline hybrid 2008 (r) & 5299618 & -10396741 & 98267 \\
\hline non-cyclic 2008 (s) & 5043234 & -10281466 & 95438 \\
\hline cyclic 2014 (s) & 7287127 & -15630858 & 138778 \\
cyclic 2014 (r) & 7490054 & -15680300 & 141143 \\
\hline hybrid 2014 (s) & 7526215 & -15647618 & 142228 \\
hybrid 2014(r) & 7896806 & -15920860 & 146535 \\
\hline non-cyclic 2014(s) & 7452564 & -15627362 & 141918 \\
non-cyclic 2014(r) & 7905615 & -15952986 & 146606 \\
\hline cyclic 2008 (p)(s) & 5142573 & -10311044 & 95396 \\
cyclic 2008 (p)(r) & 5256959 & -10399474 & 96196 \\
\hline hybrid 2008(p)(r) & 5461560 & -10564349 & 96886 \\
\hline cyclic 2014 (p)(s) & 7596212 & -15686365 & 143135 \\
cyclic 2014 (p)(r) & 7767140 & -15955037 & 143612 \\
\hline hybrid 2014 (p)(s) & 7821338 & -15855025 & 143345 \\
hybrid 2014 (p)(r) & 8229227 & -16174935 & 147373 \\
\hline non-cyclic 2014 (p)(s) & 7749755 & -15803502 & 143227 \\
non-cyclic 2014 (p)(r) & 8259557 & -16166223 & 148017 \\
\hline
\end{tabular}

Table 5: Differences between passenger satisfaction (s) and revenue (r) oriented timetable designs

passengers is more or less the same between the two concepts of the design, it proves that the somehow "capitalist" concept of revenue maximization is not anti-passenger and that it is suitable for the public operators as well. This should encourage operators to consider the new approach proposed in this research to design their timetable.

\section{Conclusion}

In this research, we further extend the passenger centric train timetabling approach with the concept of the competitive market by accounting for elasticity of the passenger demand. We include a competing operator into the market, so that the passengers are not captive in the system. This framework allows for a prediction of the realized demand, which subsequently allows to calculate the revenue of the operator. In the second phase, we integrate the proposed timetabling problem with the ticket pricing problem.

The results of our case study show that accounting for the behavioral dimension of the problem has a significant impact on the generated revenue. We show on our case study that the passenger oriented timetabling can be combined with the ticket pricing. The resulting pricing strategies suggest 
higher prices for large volumes of demand by location and time. The two together can further increase the revenue up to additional 15\%. Given that we used the best possible competitor, in our case study, the gain is expected to be even larger, when this is not the case. Lastly, we provide empirical evidence that the concept of revenue maximization is in line with passengers' objective of their satisfaction maximization. Therefore, this concept is suitable for public operators as well.

In this research, we have considered the passengers to be homogeneous. However, in the real world, this is not the case. Each person has a different behavior. This behavior is incorporated in the discrete choice model through the taste parameters beta. These parameters may vary across the population. We propose to treat this as a possible future extension. Subsequently, this would allow for an introduction of different class fares and to further improve the revenue of the operator. Moreover, many pricing estimation techniques are used by the industry, such as the zone pricing, OD pair pricing, time period pricing, etc. Based on the characteristics of the selected pricing scheme, the presented simulated annealing algorithm can be adapted. In this case, a study evaluating and generalizing the pricing system could be other future direction of this research. 


\section{Bibliography}

Armstrong, A. and Meissner, J. (2010). Railway revenue management: Overview and models (operations research), Working Papers MRG/0019, Department of Management Science, Lancaster University.

Barrena, E., Canca, D., Coelho, L. C. and Laporte, G. (2014a). Exact formulations and algorithm for the train timetabling problem with dynamic demand, Computers \& Operations Research 44: 66 74.

Barrena, E., Canca, D., Coelho, L. C. and Laporte, G. (2014b). Single-line rail rapid transit timetabling under dynamic passenger demand, Transportation Research Part B: Methodological 70: $134-150$.

Ben-Akiva, M. and Bierlaire, M. (2003). Discrete choice models with applications to departure time and route choice, Handbook of transportation science, Springer, pp. 7-37.

Ben-Akiva, M., Cascetta, E., Coppola, P., Papola, A. and Velardi, V. (2010). High speed rail demand forecasting: Italian case study, in Association for European Transport (ed.), Proceedings of the European Transport Conference, 2010, Association for European Transport.

Ben-Akiva, M. and Lerman, S. (1985). Discrete Choice Analysis, The MIT Press, Cambridge Massachusetts.

Bharill, R. and Rangaraj, N. (2008). Revenue management in railway operations: A study of the rajdhani express, indian railways, Transportation Research Part A: Policy and Practice 42(9): 1195 - 1207. Institutional Reform in Land Passenger Transport.

Börjesson, M. (2014). Forecasting demand for high speed rail, Transportation Research Part A: Policy and Practice 70: $81-92$.

Canca, D., Barrena, E., Algaba, E. and Zarzo, A. (2014). Design and analysis of demand-adapted railway timetables, Journal of Advanced Transportation 48(2): 119 -137.

Canca, D., Barrena, E., De-Los-Santos, A. and Andrade-Pineda, J. L. (2016). Setting lines frequency and capacity in dense railway rapid transit networks with simultaneous passenger assignment, Transportation Research Part B: Methodological 93, Part A: 251 - 267.

Canca, D., De-Los-Santos, A., Laporte, G. and Mesa, J. A. (2016). A general rapid network design, line planning and fleet investment integrated model, Annals of Operations Research 246(1): 127144.

Caprara, A., Fischetti, M. and Toth, P. (2002). Modeling and solving the train timetabling problem, Operations Research 50(5): 851-861.

Cordone, R. and Redaelli, F. (2011). Optimizing the demand captured by a railway system with a regular timetable, Transportation Research Part B: Methodological 45(2): 430 - 446. 
de Keizer, B., Geurs, K. and Haarsman, G. (2012). Interchanges in timetable design of railways: A closer look at customer resistance to interchange between trains., in AET (ed.), Proceedings of the European Transport Conference, Glasgow, 8-10 October 2012 (online), AET.

Espinosa-Aranda, J. L., García-Ródenas, R., del Carmen Ramírez-Flores, M., López-García, M. L. and Angulo, E. (2015). High-speed railway scheduling based on user preferences, European Journal of Operational Research 246(3): 772 - 786.

Globes (2015). Israel railways passenger traffic up 7.5\% in 2014.

URL: $\quad h t t p: / / w w w . g l o b e s . c o . i l / e n / a r t i c l e-i s r a e l-r a i l w a y s-p a s s e n g e r-t r a f f i c-u p-75-i n-2014-$ 1001005774

Hetrakul, P. and Cirillo, C. (2014). A latent class choice based model system for railway optimal pricing and seat allocation, Transportation Research Part E: Logistics and Transportation Review 61: $68-83$.

Hoppmann, H., Borndörfer, R. and Karbstein, M. (2015). Timetabling and passenger routing in public transport, Proceedings of Conference on Advanced Systems in Public Transport 2015 (CASPT2015). accepted for publication.

Jiang, X., Zhang, L. and Chen, X. M. (2014). Short-term forecasting of high-speed rail demand: A hybrid approach combining ensemble empirical mode decomposition and gray support vector machine with real-world applications in china, Transportation Research Part C: Emerging Technologies 44: 110 - 127.

Kaspi, M. and Raviv, T. (2013). Service-oriented line planning and timetabling for passenger trains, Transportation Science 47(3): 295-311.

Kirkpatrick, S., Gelatt, C. D. and Vecchi, M. P. (1983). Optimization by simulated annealing, SCIENCE 220(4598): 671-680.

Li, T., van Heck, E., Vervest, P., Voskuilen, J., Hofker, F. and Jansma, F. (2006). Passenger travel behavior model in railway network simulation, Proceedings of the 38th Conference on Winter Simulation, WSC '06, Winter Simulation Conference, pp. 1380-1387.

Luethi, M., Weidmann, U. A. and Nash, A. (2007). Passenger Arrival Rates at Public Transport Stations, 86th Annual Meeting of the Transportation Research Board.

Marín, Á. and García-Ródenas, R. (2009). Location of infrastructure in urban railway networks, Computers \& Operations Research 36(5): 1461-1477.

Niu, H. and Zhou, X. (2013). Optimizing urban rail timetable under time-dependent demand and oversaturated conditions, Transportation Research Part C: Emerging Technologies 36: 212 - 230.

Nuzzolo, A., Crisalli, U. and Gangemi, F. (2000). A behavioural choice model for the evaluation of railway supply and pricing policies, Transportation Research Part A: Policy and Practice 34(5): 395 $-404$. 
Peeters, L. (2003). Cyclic Railway Timetable Optimization, ERIM Ph.D. series Research in Management, Erasmus Research inst. of Management (ERIM).

Robenek, T., Azadeh, S. S., Maknoon, Y. and Bierlaire, M. (2017). Hybrid cyclicity: Combining the benefits of cyclic and non-cyclic timetables, Transportation Research Part C: Emerging Technologies 75: $228-253$.

Robenek, T., Maknoon, Y., Azadeh, S. S., Chen, J. and Bierlaire, M. (2016). Passenger centric train timetabling problem, Transportation Research Part B: Methodological 89: 107 - 126.

Schmidt, M. and Schöbel, A. (2015). Timetabling with passenger routing, OR Spectrum 37(1): 75-97.

Schöbel, A. (2012). Line planning in public transportation: models and methods, OR Spectrum 34: 491-510.

Serafini, P. and Ukovich, W. (1989). A mathematical model for periodic scheduling problems, SIAM J. Discret. Math. 2(4): 550-581.

Shiftan, Y., Sharaby, N. and Solomon, C. (2008). Transport project appraisal in israel, Transportation Research Record: Journal of the Transportation Research Board (2079): 136-145.

Small, K. A. (1982). The scheduling of consumer activities: Work trips, The American Economic Review 72(3): pp. 467-479.

Sparing, D. and Goverde, R. M. (2017). A cycle time optimization model for generating stable periodic railway timetables, Transportation Research Part B: Methodological 98(Supplement C): 198 $-223$.

Sun, L., Jin, J. G., Lee, D.-H., Axhausen, K. W. and Erath, A. (2014). Demand-driven timetable design for metro services, Transportation Research Part C: Emerging Technologies 46: 284 - 299.

van Vuuren, D. (2002). Optimal pricing in railway passenger transport: theory and practice in the netherlands, Transport Policy 9(2): 95 - 106.

Wang, Y., Tang, T., Ning, B., van den Boom, T. J. and Schutter, B. D. (2015). Passenger-demandsoriented train scheduling for an urban rail transit network, Transportation Research Part C: Emerging Technologies 60: $1-23$.

Wardman, M. (1997). Inter-urban rail demand, elasticities and competition in great britain: Evidence from direct demand models, Transportation Research Part E: Logistics and Transportation Review 33(1): $15-28$.

Wardman, M. (2004). Public transport values of time, Transport Policy 11(4): 363 - 377.

Wardman, M. (2006). Demand for rail travel and the effects of external factors, Transportation Research Part E: Logistics and Transportation Review 42(3): 129 - 148. 
Wardman, M., Lythgoe, W. and Whelan, G. (2007). Rail passenger demand forecasting: Crosssectional models revisited, Research in Transportation Economics 20: 119 - 152. Railroad Economics.

Whelan, G. and Johnson, D. (2004). Modelling the impact of alternative fare structures on train overcrowding, International Journal of Transport Management 2(1): 51 - 58. Rail Policy and Planning in Europe.

Yin, J., Tang, T., Yang, L., Gao, Z. and Ran, B. (2016). Energy-efficient metro train rescheduling with uncertain time-variant passenger demands: An approximate dynamic programming approach, Transportation Research Part B: Methodological 91: 178 - 210.

Yin, J., Yang, L., Tang, T., Gao, Z. and Ran, B. (2017). Dynamic passenger demand oriented metro train scheduling with energy-efficiency and waiting time minimization: Mixed-integer linear programming approaches, Transportation Research Part B: Methodological 97: 182 - 213.

Zhou, W., Tian, J., Xue, L., Jiang, M., Deng, L. and Qin, J. (2017). Multi-periodic train timetabling using a period-type-based lagrangian relaxation decomposition, Transportation Research Part B: Methodological 105(Supplement C): 144 - 173.

\section{A Data Description}

The data used in this study were obtained from the IR's website (www.rail.co.il/EN) and from other studies concerning the IR's network (Kaspi and Raviv (2013)). An algorithm in Java was coded, in order to find a set of all possible paths between every OD pair. The algorithm allows a maximum of 3 consecutive lines to get from an origin to a destination. The algorithm iterates through all OD pairs, where at first it considers the paths that consist of a single line and then the paths that would transfer from the currently selected line up to two other lines. The transfer from one line to another can be made only at one of the designated transfer points (there are 7 recommended interchange stations in the network of Israel - Figure 2). Note that the fact, that a transfer is actually possible depends on the operated timetable. Therefore, some paths might be eliminated later on by the EPCTTP model itself. When all the possible paths are generated, the algorithm removes the paths that a passenger would not consider (note that these rules are related to the network layout of Israel and might differ for other case studies):

- paths that consist of several lines including a direct line between the given OD pair, where both options travel on the same infrastructure (i.e. the passenger would rather stay on the direct line instead of transferring to another line).

- paths that consist of several lines, where two of them can reach the given destination. Changing one train to another, when both of them are going to the same destination would not make sense (the same does not happen for the origin). 
- paths that take $25 \%$ longer generalized time (sum of in-vehicle-times and transfer penalties) than the shortest possible path.

- paths that consist of redundant transfers, i.e. transferring from one line to another line that covers the same stations.

The traveling times have been extracted from the IR's website along with the dwell times at stations that remain fixed (as of the timetable 2013/14). The minimum transfer time is set to 4 minutes as in Kaspi and Raviv (2013). The Value Of Time of commuters in Israel as of the year 2012 is 21.12 New Israeli Shekel (NIS)/hour (the updated value was given to us by the author of Shiftan et al. (2008)). The $\beta$ parameters and their values are as follows: $\beta_{W}=-2.5$ (Wardman (2004)), $\beta_{\mathrm{T}}=-10$ (de Keizer et al. (2012)), $\beta_{\mathrm{E}}=-0.5$ and $\beta_{\mathrm{L}}=-1$ (Small (1982)).

\section{A.1 Passenger}

The OD flows were kindly provided by Mor Kaspi and Tal Raviv, who have cleaned the ticket selling machines' data for the year 2008 and produced the flows of an average working day in Israel. They have used this data in their study Kaspi and Raviv (2013). The OD matrix consists of hourly passenger rates between 6 a.m. and 1 a.m. The flows were smoothed into minutes by using non-homogenous Poisson process, where the hourly flows per OD pair were used as the arrival rate variable. Since the schedule passenger delay is related to the destination, we have added the time that it takes to get from an origin to a destination using the shortest path (if the path consisted of transfers, we assumed the perfect connection, i.e. only the minimum transfer time without any additional waiting at the transfer station). The size of the passenger groups were left as they were generated by the Poisson process. In total there are 1505 out of 2162 OD pairs with 126036 passengers in 2008 (with 95062 passenger groups) and 193886 passengers (with 129780 passenger groups) in 2014.

\section{A.2 Operator}

As no information about the rolling stock fleet of IR is available, we have introduced the following assumptions:

- The fleet is homogenous

- A train unit has a passenger capacity of 250

- Each train consist of 2 train units

In order to verify that the assumed train capacity is reasonable, we have solved the un-capacitated PCTTP of the IR 13/14 timetable under the 2008 demand, where the average train occupation was 172 passengers per train per segment (pptps), minimum occupation was 0 pptps, maximum occupation 1188 pptps and median was 124 pptps. Thus the capacity of 250 passengers per train unit offers a good level of service. 


\section{B Assumptions}

In this section, we organize all the assumptions of the framework. Some of the assumptions may be replaced,when additional information is available.

\section{B.1 Demand Model}

- A mode choice model is solved a priori providing the potential railway mode demand population.

- An opt-out option is needed, in order for the framework to work. The proposed generic opt-out option consist in the shortest path with zero waiting time(s) in the transfer(s) and that arrives exactly on time to the destination (no schedule passenger delay). Its fare is set to the initial fare of the optimized TOC.

- No source of additional heterogeneity outside of the error term is considered.

- The error term is independently and identically distributed according to the Extreme Value Distribution.

\section{B.2 Supply Model}

- The LPP is solved a priori and its results serve as the inputs for the EPCTTP.

- The dwell times and the running times are kept constant, only the departures from the origin stations are treated as decisions.

- The passenger groups are unsplittable. However, groups of a single passenger can be used to bypass this assumption.

- The train fleet is homogeneous, but can be adapted to reflect heterogeneity.

- The passengers are assigned according to the FCFS policy and their revenue potential.

- A segment based pricing is used. However, it can be changed to OD based pricing or to a zone system.

- The traditional TTP needs to be solved using the outputs of the EPCTTP, in order to secure the safety of the network.

\section{Mathematical Formulation and Notation}

\begin{tabular}{l|l|l|c} 
Name & Description & Units & Type \\
\hline
\end{tabular}




\begin{tabular}{|c|c|c|c|}
\hline $\mathrm{L}$ & set of operated train lines given by the LPP & - & set \\
\hline $\mathrm{V}^{\ell}$ & set of available trains for the line $\ell$ (frequency) & - & set \\
\hline$(\ell, v)$ & a train $v$ serving the line $\ell$ & - & index \\
\hline I & set of origin-destination pairs & - & set \\
\hline $\mathrm{T}_{\mathrm{i}}$ & set of desired arrival times for OD pair $i$ & - & set \\
\hline$(i, t)$ & $\begin{array}{l}\text { a passenger group traveling between OD pair } i \text { with a desired } \\
\text { arrival time } t\end{array}$ & - & index \\
\hline$P_{i}$ & set of possible paths between OD pair $i$ & - & set \\
\hline$P_{i t}$ & set of available paths for a group $(i, t)$ & - & set \\
\hline K & set of cycles within the planning horizon & - & set \\
\hline S & set of segments in the network & - & set \\
\hline$S^{\ell}$ & $\begin{array}{l}\text { ordered set of segments crossed by the line } \ell \text { to get from its } \\
\text { origin station to its destination station }\end{array}$ & - & set \\
\hline $\mathrm{H}$ & duration of the planning horizon & $\min$ & parameter \\
\hline$\beta s$ & as given in Section 3 & $\frac{1}{\min }$ & parameter \\
\hline VOT & value-of-time & $\frac{\text { monitary }}{\min }$ & parameter \\
\hline$n_{i t}$ & $\begin{array}{l}\text { number of passengers wishing to travel between OD pair } i \text { at } \\
\text { time } t\end{array}$ & - & parameter \\
\hline$a_{i t}$ & $\begin{array}{l}\text { desired arrival time of a passenger group }(i, t) \text { to its destina- } \\
\text { tion }\end{array}$ & $\min$ & parameter \\
\hline$b_{i}^{p \ell}$ & $\begin{array}{l}\text { time to get from the origin station of a train on line } \ell \text { in path } \\
p \text { to a boarding station of passengers traveling between OD } \\
\text { pair } i\end{array}$ & $\min$ & parameter \\
\hline $\mathrm{m}$ & minimum transfer time from one train to another & $\min$ & parameter \\
\hline$r_{i}^{p}$ & $\begin{array}{l}\text { total time spent on board of trains on path } p \text { between OD } \\
\text { pair } i\end{array}$ & $\min$ & parameter \\
\hline$r_{i}^{p j}$ & in-vehicle-time on train $j$ in path $p$ between OD pair $i$ & $\min$ & parameter \\
\hline $\mathrm{C}$ & maximum capacity of any train on any of its segment & - & parameter \\
\hline$u^{p}$ & is the number of transfers in the path $p$ & - & parameter \\
\hline c & size of the cycle & $\min$ & parameter \\
\hline W & train capacity & - & parameter \\
\hline$x_{\ell v}^{\mathrm{ps}}$ & 1 - if path $p$ uses train $(\ell, v)$ on segment $s, 0$ - otherwise & binary & parameter \\
\hline$\overline{\mathrm{a}}_{i \mathrm{i}}^{\mathrm{p}}$ & $\begin{array}{l}\text { actual arrival time of passenger group }(i, t) \text { to its destination } \\
\text { using path } p\end{array}$ & $\min$ & decision \\
\hline $\mathbf{o}_{\ell v}^{s}$ & occupation of a train $(\ell, v)$ on a segment $s$ & - & decision \\
\hline$f_{\ell v}^{s}$ & ticket fare of traveling on segment $s$ with a train $(\ell, v)$ & monetary & decision \\
\hline$f^{p}$ & total ticket fare of using path $p$ & monetary & decision \\
\hline $\operatorname{Pr}_{i t}^{p}$ & $\begin{array}{l}\text { probability of the passenger group }(i, t) \text { to select the path } p \\
\text { as a linear function of } w, \delta, \gamma \text { on the current timetable } d\end{array}$ & - & decision \\
\hline$d_{\ell v}$ & $\begin{array}{l}\text { the departure time of a train } v \text { on the line } \ell \text { (from its first } \\
\text { station) }\end{array}$ & $\min$ & decision \\
\hline
\end{tabular}




\begin{tabular}{|c|c|c|c|}
\hline$w_{i t}^{p}$ & waiting time of a passenger group $(i, t)$ using path $p$ & $\min$ & decision \\
\hline$w_{i t}^{p j}$ & $\begin{array}{l}\text { waiting time of passenger group }(i, t) \text { using path } p \text { while } \\
\text { transferring from train } j \text { to train } j+1\end{array}$ & $\min$ & decision \\
\hline$\delta_{i t}^{p}$ & $\begin{array}{l}\text { the schedule passenger delay of being early in path } p \text { of a } \\
\text { passenger group }(i, t)\end{array}$ & $\min$ & decision \\
\hline$\gamma_{i t}^{p}$ & $\begin{array}{l}\text { the schedule passenger delay of being late in path } p \text { of a } \\
\text { passenger group }(i, t)\end{array}$ & $\min$ & decision \\
\hline$z_{\ell v}$ & dummy variable to help modeling the cyclicity & $\mathbb{N}$ & decision \\
\hline q & 1 - if a train $(\ell, v)$ has a cyclic departure time, 0 - otherwise & binary & decision \\
\hline & $\begin{array}{l}1 \text { - if there is a cyclic train scheduled in the cycle } k \text { on the } \\
\text { line } \ell, 0 \text { - otherwise }\end{array}$ & binary & decision \\
\hline$\tau_{i t}^{p}$ & $\begin{array}{l}1-\text { if path } p \text { is included in the choice set of passenger group } \\
(i, t) ; 0 \text { - otherwise }\end{array}$ & binary & decision \\
\hline
\end{tabular}

Table 6: Mathematical notations

The EPCTTP model is formulated as follows:

$$
\begin{aligned}
& \max \sum_{\ell \in \mathrm{L}} \sum_{v \in \mathrm{V}^{\ell}} \sum_{s \in \mathrm{S}^{\ell}} \mathrm{o}_{\ell v}^{s} \cdot \mathrm{f}_{\ell v}^{\mathrm{s}}, \\
& \mathrm{o}_{\ell v}^{s}=\sum_{i \in \mathrm{I}} \sum_{\mathrm{t} \in \mathrm{T}_{i}} \sum_{p \in \mathrm{P}_{i \mathrm{t}}} x_{\ell v}^{\mathrm{ps}} \cdot \mathrm{n}_{\mathrm{it}} \cdot \operatorname{Pr}_{\mathrm{it}}^{\mathrm{p}}, \quad \forall \ell \in \mathrm{L}, \forall v \in \mathrm{V}^{\ell}, \forall s \in \mathrm{S}^{\ell}, \\
& \mathrm{o}_{\ell v}^{\mathrm{s}} \leq \mathrm{W}, \quad \forall \ell \in \mathrm{L}, \forall v \in \mathrm{V}^{\ell}, \forall \mathrm{s} \in \mathrm{S}^{\ell} \\
& \mathrm{P}_{i \mathrm{t}}=\bigcup_{\mathrm{p}=1}^{\left|\mathrm{P}_{\mathrm{i}}\right|} \mathrm{p} \cdot \tau_{\mathrm{it}}^{\mathrm{p}}, \quad \forall \mathrm{i} \in \mathrm{I}, \forall \mathrm{t} \in \mathrm{T}_{\mathrm{i}} \\
& \mathrm{f}^{\mathrm{p}}=\sum_{\mathrm{j} \in \mathrm{J}^{\mathrm{p}}} \sum_{s \in \mathrm{S}^{\ell_{j}}} \mathrm{f}_{\ell_{\mathrm{j}} v_{\mathrm{j}}}^{\mathrm{s}}, \quad \forall \mathrm{i} \in \mathrm{I}, \forall \mathrm{t} \in \mathrm{T}_{\mathrm{i}}, \forall \mathrm{p} \in \mathrm{P}_{\mathrm{it}}, \\
& \mathrm{d}_{\ell v} \leq \mathrm{d}_{\ell v+1}-1, \quad \forall \ell \in \mathrm{L}, \forall v \in \mathrm{V}^{\ell}: v<\left|\mathrm{V}^{\ell}\right| \text {, } \\
& \mathrm{d}_{\ell v} \in \mathbb{N}, \quad \forall \ell \in \mathrm{L}, \forall v \in \mathrm{V}^{\ell} \text {, } \\
& \mathrm{f}_{\ell v}^{s} \geq 0, \quad \forall \ell \in \mathrm{L}, \forall v \in \mathrm{V}^{\ell}, \forall \mathrm{s} \in \mathrm{S}^{\ell}, \\
& \mathrm{f}^{\mathrm{p}} \geq 0, \quad \forall \mathrm{i} \in \mathrm{I}, \forall \mathrm{t} \in \mathrm{T}_{\mathrm{i}}, \forall \mathrm{p} \in \mathrm{P}_{\mathrm{it}}, \\
& \mathrm{o}_{\ell v}^{s} \geq 0, \quad \forall \ell \in \mathrm{L}, \forall v \in \mathrm{V}^{\ell}, \forall \mathrm{s} \in \mathrm{S}^{\ell}, \\
& \tau_{i t}^{p} \in(0,1), \quad \forall i \in I, \forall t \in T_{i}, \forall p \in P_{i t}, \\
& \operatorname{Pr}_{i t}^{p} \geq 0, \quad \forall i \in I, \forall t \in T_{i}, \forall p \in P_{i t},
\end{aligned}
$$

Objective function (11) is maximizing the TOC's revenue that is given as the train occupation $\mathrm{o}_{\ell v}^{s}$ of each train $(\ell, v)$ on each of its segments multiplied by the fare cost $f_{\ell v}^{s}$ of train $(\ell, v)$ on segment $s$. Constraints (12) use the logit model to estimate the train occupation. The parameter $\chi_{\ell v}^{p s}$ indicates 
whether path $p$ is using train $(\ell, v)$ on segment $s$ (equals to 1 ) or not (equals to 0). Constraints (13) secure that the capacity of a train is not exceeded at any point of its travel. If a train is full at some of the segments, all the paths of any additional passengers using that train at that segment are removed from their choice sets inside of the logit model. Note, that a simple multiplication by the decision variable $\tau_{i t}^{p}$ would create a proper behavior within the optimization model, but would lead to wrong predictions of the probabilities. Therefore, constraints (14) explicitly define those paths that are to be included in the set $P_{i t}$. The decision itself, on which passenger should be restricted, is driven by the objective function. Constraints (15) calculate the fare to be payed for usage of each path $p$. Constraints (16) remove the symmetrical solutions, i.e. trains are ordered according to their departure times (ascending). The constraints (17)-(22) are the domain constraints.

Additional constraints, modeling some of the attributes used in the observable part of the utility function, are needed:

$$
\begin{aligned}
w_{i t}^{p}=\sum_{j \in J^{p: j<\left|J^{p}\right|}} w_{i t}^{p j}, & \forall i \in I, \forall t \in T_{i}, \forall p \in P_{i t}, \\
w_{i t}^{p j}=\left(d_{\ell_{j+1} v_{j+1}}+b_{i}^{p j+1}\right) & \forall i \in I, \forall t \in T_{i}, \forall p \in P_{i t}, \\
-\left(d_{\ell_{j} v_{j}}+b_{i}^{p j}+r_{i}^{p j}+m\right), & \forall j \in J^{p}: j<\left|J^{p}\right|, \\
\delta_{i t}^{p} \geq a_{i t}-\bar{a}_{i t}^{p}, & \forall i \in I, \forall t \in T_{i}, \forall p \in P_{i t}, \\
\gamma_{i t}^{p} \geq \bar{a}_{i t}^{p}-a_{i t}, & \forall i \in I, \forall t \in T_{i}, \forall p \in P_{i t}, \\
\bar{a}_{i t}^{p}=\left(d_{\ell_{\mid j p}\left|v_{\mid j} p\right|}+b_{i}^{p\left|j^{p}\right|}+r_{i}^{p\left|J^{p}\right|}\right), & \forall i \in I, \forall t \in T_{i}, \forall p \in P_{i t}, \\
w_{i t}^{p} \geq 0, & \forall i \in I, \forall t \in T_{i}, \forall p \in P_{i t}, \\
w_{i t}^{p j} \geq 0, & \forall i \in I, \forall t \in T_{i}, \forall p \in P_{i t}, \\
\delta_{i t}^{p} \geq 0, & \forall i \in I, \forall t \in T_{i}, \forall p \in P_{i t}, \\
\gamma_{i t}^{p} \geq 0, & \forall i \in I, \forall t \in T_{i}, \forall p \in P_{i t}, \\
\bar{a}_{i t}^{p} \geq 0, & \forall i \in I, \forall t \in T_{i}, \forall p \in P_{i t} .
\end{aligned}
$$

Constraints (23) add up the waiting times in all of the transfer stations in path $p$. Subsequently, constraints (24) define the value of the waiting time in the transferring stations. These constraints subtract the arrival time (departure time from its origin station plus the time to get to the boarding station of the passenger plus the time to get to the transfer station) of the origin train at the transferring station plus the necessary minimum transfer time $m$, from the departure time of the destination train at the transferring station (departure time from its origin station plus the time to get to the transfer station). Note, that the paths where a transfer is not possible will be removed by constraints (14). Constraints (25) calculate the early schedule passenger delay and the constraints (26) calculate the late schedule passenger delay. The actual arrival time of a passenger group to its destination is given by constraints (27). Constraints (28)-(32) are the domain constraints. 